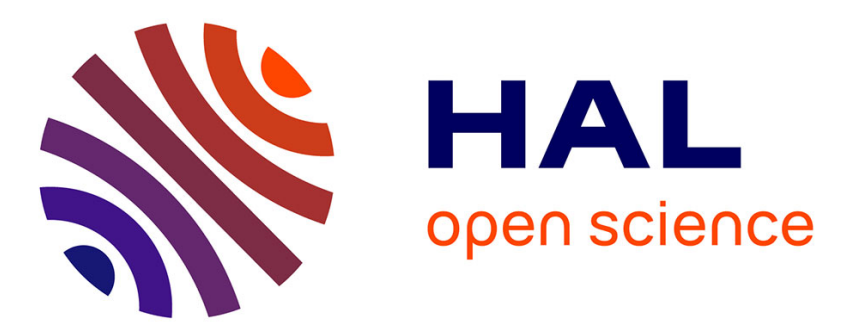

\title{
Sequential parameter estimation for fluid-structure problems. Application to hemodynamics
}

\author{
Cristobal Bertoglio, Philippe Moireau, Jean-Frédéric Gerbeau
}

\section{To cite this version:}

Cristobal Bertoglio, Philippe Moireau, Jean-Frédéric Gerbeau. Sequential parameter estimation for fluid-structure problems. Application to hemodynamics. International Journal for Numerical Methods in Biomedical Engineering, 2012, 28 (4), pp.434-455. 10.1002/cnm.1476 . inria-00603399

\section{HAL Id: inria-00603399 \\ https://hal.inria.fr/inria-00603399}

Submitted on 24 Jun 2011

HAL is a multi-disciplinary open access archive for the deposit and dissemination of scientific research documents, whether they are published or not. The documents may come from teaching and research institutions in France or abroad, or from public or private research centers.
L'archive ouverte pluridisciplinaire HAL, est destinée au dépôt et à la diffusion de documents scientifiques de niveau recherche, publiés ou non, émanant des établissements d'enseignement et de recherche français ou étrangers, des laboratoires publics ou privés. 


\title{
$\operatorname{IN} R \operatorname{IA}$
}

INSTITUT NATIONAL DE RECHERCHE EN INFORMATIQUE ET EN AUTOMATIQUE

\section{Sequential parameter estimation for fluid-structure problems. Application to hemodynamics}

\author{
Cristóbal Bertoglio — Philippe Moireau — Jean-Frederic Gerbeau
}

\section{$\mathbf{N}^{\circ} \mathbf{7 6 5 7}$}

Juin 2011

Observation, Modeling, and Control for Life Sciences

apport

de recherche 



\title{
Sequential parameter estimation for fluid-structure problems. Application to hemodynamics
}

\author{
Cristóbal Bertoglio, Philippe Moireau, Jean-Frederic Gerbeau \\ Theme : Observation, Modeling, and Control for Life Sciences \\ Computational Sciences for Biology, Medicine and the Environment \\ Équipes-Projets REO et MACS \\ Rapport de recherche $\mathrm{n}^{\circ} 7657$ - Juin 2011 - 32 pages
}

\begin{abstract}
We present a robust and computationally efficient parameter estimation strategy for fluid-structure interaction problems. The method is based on a filtering algorithm restricted to the parameter space, known as the reduced order Unscented Kalman Filter [23. It does not require any adjoint or tangent problems. In addition, it can easily be run in parallel, which is of great interest in fluid-structure problems where the computational cost of the forward simulation is already a challenge in itself. We illustrate our methodology with the estimation of the artery wall stiffness from the wall displacement measurements - as they could be extracted from medical imaging - in a three-dimensional idealized abdominal aortic aneurysm. We also show preliminary results about the estimation of the proximal Windkessel resistance, which is an important parameter for setting appropriate fluid boundary conditions.
\end{abstract}

Key-words: Inverse problem, fluid-structure interaction, reduced order Unscented Kalman Filter, arterial stiffness estimation 


\section{Estimation de paramètres séquentielle pour des problèmes fluide-structure. Application à l'hémodynamique}

Résumé : Nous proposons une stratégie d'estimation de paramètres robuste et efficace pour des problèmes d'interaction fluide-structure. La méthode est basée sur un algorithme de filtrage limité à l'espace des paramètres, connue sous le nom de Filtre de Kalman réduit [23]. Il ne nécessite aucun adjoint ou problème tangent. De plus, il peut facilement être exécuté en parallèle, ce qui est d'un grand intérêt pour les problèmes d'interaction fluide-structure, où le coût de calcul de la simulation directe est déjà un défi en soi. Nous illustrons notre méthodologie avec l'estimation de la rigidité des artères à partir de mesures de déplacements de la paroi - tels qu'ils pourraient être extraits de l'imagerie médicale - dans un anévrisme de l'aorte abdominale idéalisée en trois dimensions. Nous montrons aussi des résultats préliminaires pour l'estimation de la résistance proximale de Windkessel, qui est un paramètre important pour les conditions aux limites du fluide.

Mots-clés : Problèmes inverses, interaction fluide-structure, Unscented Kalman Filter, estimation de la raideur des artères 


\section{Introduction}

During the last decade, many works have been devoted to fluid-structure interaction (FSI) in large arteries. Important progress has been made to improve the robustness and the efficiency of the coupling algorithms (see for example 9] and the references therein). But to make the FSI models useful in the clinical context, they have to be personalized, i.e. adapted to the patient. This requires not only to get the patient anatomy, but also to fit the model parameters using the available clinical measurements. The problem of reducing the uncertainties of a model using partial observations of the state variables is well-known in many fields of science and engineering and is referred to as data assimilation.

The purpose of this article is to present a data assimilation strategy for FSI problems. We apply it on an academical - but realistic - test case of blood flow to demonstrate its capability to recover the artery wall stiffness from the displacements of the wall.

Data assimilation of distributed mechanical systems can be based on a variational approach (see e.g. [1, 5]) by minimizing a least square criterion which includes a regularization term and the difference between the observations and the model prediction. One of the main difficulties of this approach lies in the iterative evaluation of the criterion, involving many solutions of the forward problem, and its gradient - typically adjoint-based - which usually requires a laborious implementation. In FSI for hemodynamics, this approach has been investigated for one-dimensional models in [19, 21, and for three-dimensional problems in [6] by modifying the minimization problem in order to avoid the resolution of the adjoint equations.

In this work, we consider a sequential approach, based on a generalization of the Kalman filter. With sequential algorithms, the model prediction is improved at every time instant by analyzing the discrepancy between the actual measurements and the model observation outputs[2, 28]. In fact, in the fully linear case, it can be proved that the Kalman filter gives the same result as a variational approach based on a least squares criterion.

Even though full Kalman filter is not tractable for distributed systems, some effective sequential procedures for mechanical systems have been recently introduced: for the state estimation [24, 25], and for the parameter identification [24, 23]. In particular, in [23], the authors formulate a reduced-order version of the Unscented Kalman Filter (UKF) [15] inspired from the Singular Evolutive Extended Kalman filter (SEIK) proposed in [30] and apply it for characterizing solid mechanics constitutive parameters. Here, we investigate the derivation of this filter in order to estimate the parameters of a FSI system. With respect to other approaches, this algorithm does not need adjoint or tangent operators and can be very easily parallelized. As a result, the total CPU time needed for the data assimilation is of the same order of magnitude as the CPU time needed for one forward simulation and the implementation does not need important modifications in existing solvers. Preliminary results of the present work have been presented in [4].

The rest of this paper is organized as follows. Section 2 introduces the FSI equations and the data assimilation problem. Section 3 is devoted to an introduction to data assimilation techniques, geared to readers with no background in this field. In particular, a short derivation of the standard Kalman filter and some extensions to nonlinear cases are presented. The purpose of these sections 
is to provide the reader with a comprehensive overview of the reduced-order Uncented Kalman filter whereas Section 4 points out the specific issues encountered in the derivation of the filtering strategy in the fluid-structure context. Finally, numerical investigations are presented in Section 5

\section{Problem setting}

\section{$2.1 \quad$ Fluid-Structure model}

We consider the mechanical interaction between an incompressible fluid and an elastic structure. The fluid is described by the Navier-Stokes equations, in a moving domain $\Omega_{\mathrm{f}}(t) \subset \mathbb{R}^{d}, d=2,3$, in an Arbitrary Lagrangian Eulerian (ALE) formulation, and the structure by the linear elasticity equations in $\Omega_{\mathrm{s}}(t) \subset \mathbb{R}^{d}$. The fluid-structure interface is denoted by $\Sigma \stackrel{\text { def }}{=} \partial \Omega_{\mathrm{s}} \cap \partial \Omega_{\mathrm{f}}$ and $\partial \Omega_{\mathrm{f}}=\Gamma^{\mathrm{in}} \cup \Gamma^{\text {out }} \cup \Sigma, \partial \Omega_{\mathrm{s}}=\Gamma^{\mathrm{d}} \cup \Gamma^{\mathrm{n}} \cup \Sigma$, are given partitions of the fluid and solid boundaries, respectively (see Figure 1). The coupled FSI problem reads as follows: find the fluid velocity $\boldsymbol{u}(t): \Omega_{\mathrm{f}}(t) \rightarrow \mathbb{R}^{d}$, the fluid pressure $p(t): \Omega_{\mathrm{f}}(t) \rightarrow \mathbb{R}$ and the structure displacement $\boldsymbol{y}(t): \Omega_{\mathrm{s}} \rightarrow \mathbb{R}^{d}$ such that

- Fluid equations:

$$
\left\{\begin{aligned}
\rho_{\mathrm{f}} \frac{\partial \boldsymbol{u}}{\partial t}{ }_{\mid \widehat{\boldsymbol{x}}}+\rho_{\mathrm{f}}\left(\boldsymbol{u}-\boldsymbol{w}_{\mathrm{f}}\right) \cdot \boldsymbol{\nabla} \boldsymbol{u}-2 \mu \boldsymbol{\nabla} \cdot \boldsymbol{\epsilon}(\boldsymbol{u})+\boldsymbol{\nabla} p=\mathbf{0}, & \text { in } \quad \Omega_{\mathrm{f}}(t) \\
\boldsymbol{\nabla} \cdot \boldsymbol{u}=0, & \text { in } \quad \Omega_{\mathrm{f}}(t) \\
\boldsymbol{u}=\boldsymbol{u}_{\mathrm{in}}, & \text { on } \quad \Gamma^{\mathrm{in}} \\
\boldsymbol{\sigma}(\boldsymbol{u}, p) \boldsymbol{n}=-\bar{p} \boldsymbol{n}, & \text { on } \quad \Gamma^{\text {out }}
\end{aligned}\right.
$$

- Structure equations:

$$
\left\{\begin{array}{rcc}
\rho_{\mathrm{s}} \partial_{t} \widehat{\boldsymbol{w}}-\eta_{\mathrm{s}} \boldsymbol{\nabla} \cdot \boldsymbol{\sigma}_{\mathrm{s}}(\widehat{\boldsymbol{w}})-\boldsymbol{\nabla} \cdot \boldsymbol{\sigma}_{\mathrm{s}}(\widehat{\boldsymbol{y}})=\mathbf{0}, & \text { in } \widehat{\Omega}_{\mathrm{s}}, \\
\widehat{\boldsymbol{w}}=\partial_{t} \widehat{\boldsymbol{y}}, & \text { in } \quad \widehat{\Omega}_{\mathrm{s}}, \\
\widehat{\boldsymbol{y}}=\mathbf{0}, & \text { on } \quad \Gamma^{\mathrm{d}}, \\
\boldsymbol{\sigma}_{\mathrm{s}}(\widehat{\boldsymbol{y}}) \boldsymbol{n}_{\mathrm{s}}=\mathbf{0}, & \text { on } \quad \Gamma^{\mathrm{n}},
\end{array}\right.
$$

- Coupling conditions:

$$
\left\{\begin{aligned}
\widehat{\boldsymbol{y}}_{\mathrm{f}}=\operatorname{Ext}\left(\widehat{\boldsymbol{y}}_{\mid \widehat{\Sigma}}\right), \quad \boldsymbol{w}_{\mathrm{f}}=\partial_{t} \widehat{\boldsymbol{y}}_{\mathrm{f}}, \quad \Omega_{\mathrm{f}}(t)=\underbrace{\left(I_{\widehat{\Omega}_{\mathrm{f}}}+\widehat{\boldsymbol{y}}_{\mathrm{f}}\right)}_{\widehat{\mathcal{A}}}\left(\widehat{\Omega}_{\mathrm{f}}, t\right), \\
\boldsymbol{u}=\boldsymbol{w}_{\mathrm{f}}, \quad \text { on } \quad \Sigma(t), \\
\boldsymbol{\sigma}_{\mathrm{s}}(\widehat{\boldsymbol{y}}) \widehat{\boldsymbol{n}}_{\mathrm{s}}+\widehat{J}_{\mathrm{f}} \widehat{\boldsymbol{\sigma}(\boldsymbol{u}, p)} \widehat{\boldsymbol{F}}_{\mathrm{f}}^{-T} \widehat{\boldsymbol{n}}_{\mathrm{f}}=\mathbf{0}, \quad \text { sur } \widehat{\Sigma},
\end{aligned}\right.
$$

with $\widehat{\boldsymbol{F}}_{\mathrm{f}}$ the deformation gradient and $\widehat{J}_{\mathrm{f}}=\operatorname{det} \widehat{\boldsymbol{F}}_{\mathrm{f}}$. Note that the equalities $\boldsymbol{u}=\boldsymbol{w}_{\mathrm{f}}$ on $\Sigma(t)$ and $\widehat{\boldsymbol{y}}_{\mathrm{f}}=\operatorname{Ext}\left(\widehat{\boldsymbol{y}}_{\mid \widehat{\Sigma}}\right)$ could be limited to the normal components (whereas the continuity of all the components of the fluid and solid velocities, $\boldsymbol{u}$ and $\boldsymbol{w}$, has of course to be enforced on $\Sigma(t))$. This problem has to be completed 


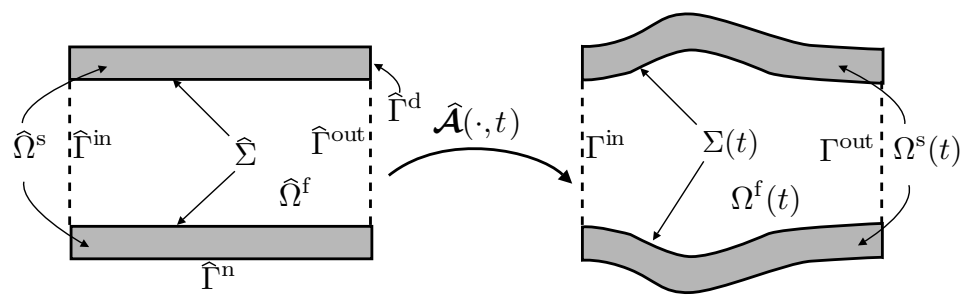

Figure 1: Reference (left) and current domain (right) in the ALE formulation.

with appropriate initial conditions: velocity $\boldsymbol{u}^{0}$ and domain displacement $\widehat{\boldsymbol{y}}_{\mathrm{f}}^{0}$ for the fluid, initial velocity $\widehat{\boldsymbol{w}}^{0}$ and displacement $\widehat{\boldsymbol{y}}^{0}$ for the solid.

In the hemodynamics problems considered in the work, the outlet pressure $\bar{p}$ is obtained by solving an ordinary differential equation coupled to the threedimensional problem:

$$
\left\{\begin{aligned}
C \frac{d \pi}{d t}+\frac{\pi-P_{V}}{R_{d}} & =Q \\
\left.\pi\right|_{t=0} & =\pi_{0} \\
\bar{p} & =\pi+R_{p} Q \\
Q & =\int_{\Gamma \text { out }} \boldsymbol{u} \cdot \boldsymbol{n}_{f},
\end{aligned}\right.
$$

where the venous pressure $P_{V}$, the distal resistance $R_{d}$, the proximal resistance $R_{p}$ and the capacitance $C$ are assumed to be given. This "zero-dimensional" equation is known as the three-element Windkessel model (see for example [12]). Note that the pressure $\pi$ belongs to the state variables of the problem. In realistic configurations, several outlets have to be considered. In such a case, there are as many systems (4) as outlets (see for example [26] for FSI in a patient-specific aorta).

\subsection{Data assimilation problem}

A physical system like blood flowing in a compliant artery can be observed through various measurement modalities: artery wall movements obtained from 4D medical imaging - computed tomography (CT), magnetic resonance imaging (MRI) or ultrasound (US) - cross section blood flow rates by Phase Contrast MRI or US, pressure in few points provided by a catheter, etc. These measurements are usually limited to a few locations in space, and sometimes come from different cardiac cycles (in this case they are resynchronized with the electrocardiogram). They are of course subject to noise and their postprocessing can introduce some further inaccuracies. Moreover, only a limited number of physical quantities can be simultaneously obtained.

The physical system of interest can also be modeled by equations like those presented in the previous section. In that case, many quantities - displacement, velocity, pressure, stress - are available in "all" locations and at "any" time instant. But of course, the model itself contains approximations - due for example to the modeling choices and the numerical solution - and relies on parameters that are not perfectly known. 
The objective of data assimilation is to take advantage of both measurements and models. Measurements can be used to reduce the uncertainties of the model, and the model can be used to access some "hidden" physical quantities, for example the mechanical stress in the artery wall. Data assimilation can also be viewed as a way to reduce the measurements noise by means of a model that takes into account the underlying physical principles.

The real state variables of the physical system of interest (for example displacements, velocities, etc.) are denoted by $x(t)$, and the parameters of the model by $\theta \in \mathbb{R}^{p}$. From now, it is assumed that the problem has already been discretized in space and we denote the semi-discrete state $X(t) \in \mathbb{R}^{N}$. The state variable is solution of the dynamical system:

$$
\dot{X}=A(X, \theta), X(0)=X_{0}
$$

In this study $X(t)$ typically denotes the finite element approximation of the displacements and the velocities in the fluid and the solid, and $\theta$ denotes for example the Young's modulus in different regions of the artery wall. So the orders of magnitude of $N$ and $p$ are typically a few hundreds of thousands and a dozen respectively. A discontinuous representation of the parameters is chosen here for the sake of simplicity but higher order discretization can also be considered 31].

The measurements $\mathcal{Z}(t)$ are defined by an observation operator $\mathcal{H}(t)$ applied to the real state $x(t)$. Assuming that an approximation $X_{n}$ of $x(n \Delta t)$ and an approximation $H_{n}$ of $\mathcal{H}(n \Delta t)$ are available, the discrete measurement vector $Z_{n} \in \mathbb{R}^{m}$ - supposed to be known - is given by:

$$
Z_{n}=H_{n}\left(X_{n}\right)+\zeta_{n}^{Z}
$$

where $\zeta_{n}^{Z}$ represents the noise of the measurement device as well as the inaccuracy resulting from the discretizations. These measurements are assumed to be available at every simulation time step. When this is not the case, they are given by a linear interpolation in time.

Data assimilation techniques usually consists of minimizing a cost function like:

$$
J(X, \theta)=\int_{0}^{T}\|Z-H(X)\|_{W^{-1}}^{2} \mathrm{~d} t+\left\|\theta-\hat{\theta}_{0}\right\|_{\left(P_{0}^{\theta}\right)^{-1}}^{2}+\left\|X(0)-\hat{X}_{0}\right\|_{\left(P_{0}^{X}\right)^{-1}}^{2},
$$

with $X$ satisfying (5). In this expression, $\hat{X}_{0}$ and $\hat{\theta}_{0}$ are given a priori values for the initial condition and parameters, and $\|\cdot\|_{W^{-1}},\|\cdot\|_{\left(P_{0}^{X}\right)^{-1}}$ and $\|\cdot\|_{\left(P_{0}^{\theta}\right)^{-1}}$ denote some norms used to measure the observations, the state and the parameters, respectively. These norms allow to give a different weight to the different terms and therefore account for the "confidence" in the different quantities. From a statistical viewpoint, the "confidence" can be viewed as the inverse of covariance matrices $\left(W, P_{0}^{X}\right.$ and $\left.P_{0}^{\theta}\right)$, which explains the notation.

Defining $\zeta^{X}=X_{0}-\hat{X}_{0}, \zeta^{\theta}=\theta-\hat{\theta}_{0}$, the optimization problem reads (keeping the same notation $J$ for the cost function):

$$
J\left(\zeta^{X}, \zeta^{\theta}\right)=\int_{0}^{T}\|Z-H(X)\|_{W^{-1}}^{2} \mathrm{~d} t+\left\|\zeta^{\theta}\right\|_{\left(P_{0}^{\theta}\right)^{-1}}^{2}+\left\|\zeta^{X}\right\|_{\left(P_{0}^{X}\right)^{-1}}^{2}
$$


This minimization problem can be addressed by many techniques that are classically divided in two groups: the variational and the sequential approaches. The variational approach consists of minimizing this cost function by an optimization algorithm that is usually based on the computation of its gradient, obtained by solving an adjoint model. The sequential approach, also known as filtering, modifies the forward dynamics with a correction term that takes into account the discrepancy between actual measurements and observations generated by the model:

$$
\dot{\hat{X}}=A(\hat{X}, \theta)+K(Z-H(\hat{X}))
$$

where $\hat{X}$ is called estimator of $X$. The quantity $Z-H(\hat{X})$ is known as the innovation, and the operator $K$ depends on the method. For linear problems, the most famous sequential approach is the Kalman filter. On a given time interval $[0, T]$, and if all the operators are linear, the variational method and the Kalman filter algorithm turn out to give the same estimation at $t=T$. Concerning the computational complexity, whereas the variational method has to solve several forward and adjoint problems on the whole interval $[0, T]$, the estimation in the sequential algorithm is computed by solving only once the filtered dynamics (7). However, the optimal operator $K$ is determined by operations (multiplications, inversions, etc.) involving full matrices of the size of the state and the observations, which makes Kalman-based filters prohibitive for discrete problems derived from partial differential equations (PDEs).

Fortunately, several alternatives exist to reduce the computational cost of filtering approaches. For instance, in [24] the authors have designed a Luenberger observer 20] for the solid mechanics state variables in order to obtain a state filter operator that is tractable. Then, the remaining uncertainties - mostly the parameters in their context - can be handled by the use of a reduced-order Kalman filter on the system corrected by the Luenberger filter [23, 24]. Our purpose in this paper is to restrict our investigation to that last part by deriving the reduced-order Uncented Kalman Filter (UKF) proposed in [23] for FSI problems. In this context, if the usual state variables arising from the PDEs discretization are assumed to be stable, the remaining uncertainties are the parameters. Nevertheless, in Section 5 , it will be shown that the Windkessel pressure, which is a state variable, can easily and advantageously be added to the set of filtered quantities.

In the next section, we give an informal introduction to some sequential methods. We start with the least squares estimation in a static case, and we gradually enrich the method until the reduced UKF algorithm that will be later applied to three-dimensional FSI inverse problem.

\section{Kalman filtering in a nutshell}

The material presented in this section is standard in many fields, like weather forecasting or oceanography. Since it is less common in mechanics, we thought it might be useful to present it in order to make the article as self-contained as possible. For a more detailed review, we refer the reader to [17, 28] among many others. 


\subsection{Static linear case: least squares estimation}

To start with, assume that there is no dynamics, that the parameters are perfectly known and that the observation operator is linear. Assume that a "guess" $\hat{X}^{-}$is available for the state, for example provided by a model, associated with a confidence matrix $\left(P^{-}\right)^{-1}$. Assume also that an observation $Z=H X+$ $\zeta^{Z}$ is available. Since $\zeta^{Z}$ is unknown, we also associate a confidence matrix $W^{-1}$ with the observations.

A quantity accounting for $\hat{X}^{-}$and $Z$, with their respective levels of confidence, can simply be obtained by minimizing the quadratic cost function:

$$
J(\hat{X})=\frac{1}{2}\left(\hat{X}-\hat{X}^{-}\right)^{T}\left(P^{-}\right)^{-1}\left(\hat{X}-\hat{X}^{-}\right)+\frac{1}{2}(Z-H \hat{X})^{T} W^{-1}(Z-H \hat{X}) .
$$

An elementary computation shows that the minimizer of $J$ is given by

$$
\hat{X}^{+}=\hat{X}^{-}+K\left(Z-H \hat{X}^{-}\right)
$$

where

$$
K=P^{+} H^{T} W^{-1}
$$

is the Kalman matrix and $P^{+}=\left(\left(P^{-}\right)^{-1}+H^{T} W^{-1} H\right)^{-1}$. Applying the Woodbury matrix inversion lemma to $P^{+}$, the Kalman matrix can also be written as

$$
K=P^{X Z}\left(P^{Z}\right)^{-1}
$$

with

$$
P^{X Z}=P^{-} H^{T} \quad \text { and } \quad P^{Z}=W+H P^{-} H^{T} .
$$

This result can also be obtained with statistical arguments considering the unknown state $X$ as a random variable. Assuming that $\hat{X}^{-}=\mathbb{E}(X)$ and that the error on the guess has a covariance matrix $P^{-}=\mathbb{E}\left(\left(X-\hat{X}^{-}\right)\left(X-\hat{X}^{-}\right)^{T}\right)$ (which is the inverse of the above "confidence matrix"). Then, looking for an $\hat{X}$ such that $\hat{X}=\mathbb{E}(X)$ and such that the trace of the associated covariance matrix is minimized, it can be shown that the result $\hat{X}^{+}$is again given by (8). In what follows, we will adopt this statistical point of view.

It will be useful to note that the covariance of $\hat{X}^{+}$is given by $P^{+}=(I-$ $K H) P^{-}$or

$$
P^{+}=P^{-}-P^{X Z}\left(P^{Z}\right)^{-1}\left(P^{X Z}\right)^{T} .
$$

\subsection{Dynamic linear case: the Kalman filter}

Now assume that the state variable satisfies a linear dynamics, without any model uncertainties:

$$
\dot{X}=A X+F
$$

and with an uncertain initial guess $X(0)=\hat{X}_{0}+\zeta^{X}\left(\hat{X}_{0}\right.$ being given and $\zeta^{X}$ unknown). After time discretization, the dynamics reads:

$$
X_{n+1}=A_{n+1} X_{n}+F_{n+1}
$$

Suppose that the following observations are available:

$$
Z_{n}=H_{n} X_{n}+\zeta_{n}^{Z}
$$


where $\zeta_{n}^{Z}$ includes the measurement noise and the discretization error.

The linear Kalman filter [18] can simply be presented as follows. Assume that $\hat{X}_{n}^{+}$is known with a covariance $P_{n}^{+}$. First, the model is used to compute a prediction:

$$
\hat{X}_{n+1}^{-}=A_{n+1} \hat{X}_{n}^{+}+F_{n+1}
$$

By linearity of $A_{n+1}$, the covariance $P_{n+1}^{-}=\mathbb{E}\left(\left(X_{n+1}-\hat{X}_{n+1}^{-}\right)\left(X_{n+1}-\hat{X}_{n+1}^{-}\right)^{T}\right)$ is given by

$$
P_{n+1}^{-}=A_{n+1} P_{n}^{+} A_{n+1}^{T} .
$$

Then, repeating mutatis mutandis the least squares argument of the case without dynamics, a correction taking into account the observation is given by:

$$
\hat{X}_{n+1}^{+}=\hat{X}_{n+1}^{-}+K_{n+1}\left(Z_{n+1}-H_{n+1} \hat{X}_{n+1}^{-}\right) .
$$

with

$$
K_{n+1}=P_{n+1}^{X Z}\left(P_{n+1}^{Z}\right)^{-1}
$$

where $P_{n+1}^{X Z}=P_{n+1}^{-} H_{n+1}^{T}$ and $P_{n+1}^{Z}=W_{n+1}+H_{n+1} P_{n+1}^{-} H_{n+1}^{T}$. As in the static case, the covariance associated with $\hat{X}_{n+1}^{+}$is given by

$$
P_{n+1}^{+}=P_{n+1}^{-}-P_{n+1}^{X Z}\left(P_{n+1}^{Z}\right)^{-1}\left(P_{n+1}^{X Z}\right)^{T} .
$$

Thus, we can iterate and perform the prediction step at the new time step $n+1$.

It can be proved that these estimations of the state and covariances can also be obtained by minimizing the functional

$$
J\left(\zeta^{X}\right)=\frac{1}{2}\|\zeta\|_{\left(P_{0}^{-}\right)^{-1}}^{2}+\frac{1}{2} \sum_{k=0}^{n+1}\left\|Z_{k}-H X_{k}\right\|_{W_{k}^{-1}}^{2}
$$

under the constraint (13). In other words, the Kalman filter estimator $\hat{X}_{n+1}^{+}$is equal to $X_{n+1}\left(\bar{\zeta}^{X}\right)$, where $\bar{\zeta}^{X}$ is the minimizer of the variational problem on $\left[0, t^{n+1}\right]$.

\subsection{Extensions to nonlinear cases}

The Kalman filter presented above is only valid when the dynamics and the observation operator are linear. To address the fluid-structure problems considered in this work, it is necessary to extend the algorithm to nonlinear cases.

The most straightforward extension consists of applying steps 16,18 and (19) with the tangent operators $A_{n+1}^{\prime} \stackrel{\text { def }}{=} A_{n+1}^{\prime}\left(\hat{X}_{n}^{+}\right)$and $H_{n+1}^{\prime} \stackrel{\text { def }}{=} H_{n+1}^{\prime}\left(\hat{X}_{n+1}^{-}\right)$, and steps (15) and (17) with the nonlinear operators $A_{n+1}(\cdot)$ and $H_{n+1}(\cdot)$ :

$$
\left\{\begin{array}{l}
\hat{X}_{n+1}^{-}=A_{n+1}\left(\hat{X}_{n}^{+}\right) \\
P_{n+1}^{-}=A_{n+1}^{\prime} P_{n}^{+}\left(A^{\prime}\right)^{T} \\
P_{n+1}^{X Z}=P_{n+1}^{-}\left(H^{\prime}\right)^{T}, P_{n+1}^{Z}=W_{n+1}+H^{\prime} P_{n+1}^{-}\left(H_{n+1}^{\prime}\right)^{T} \\
\hat{X}_{n+1}^{+}=\hat{X}_{n+1}^{-}+K_{n+1}\left(Z_{n+1}-H_{n+1}\left(\hat{X}_{n+1}^{-}\right)\right), K_{n+1}=P_{n+1}^{X Z}\left(P_{n+1}^{Z}\right)^{-1} \\
P_{n+1}^{+}=P_{n+1}^{-}-P_{n+1}^{X Z}\left(P_{n+1}^{Z}\right)^{-1}\left(P_{n+1}^{X Z}\right)^{T}
\end{array}\right.
$$


But the resulting algorithm, called Extended Kalman Filter (EKF), has two drawbacks: the computation of the tangent operators and the precision of the estimated values.

To understand the latter issue, it is interesting to consider how the mean value of a random variable is transported by a nonlinear operator $\mathcal{A}$. Let $X$ denote a random variable in $\mathbb{R}^{N}$, its mean value $\hat{X}=\mathbb{E}(X)$ and its covariance $\mathcal{P}=\mathbb{E}\left((X-\hat{X})(X-\hat{X})^{T}\right)$. A Taylor expansion around $\hat{X}$ gives:

$Y \stackrel{\text { def }}{=} \mathcal{A}(X)=\mathcal{A}(\hat{X})+\mathcal{A}^{\prime}(\hat{X}) \cdot(X-\hat{X})+\frac{1}{2}(X-\hat{X})^{T} \cdot \mathcal{A}^{\prime \prime}(\hat{X}) \cdot(X-\hat{X})+\mathcal{O}\left(|X-\hat{X}|^{3}\right)$

Hence, the propagated mean is given by

$$
\mathbb{E}(Y)=\mathcal{A}(\hat{X})+\frac{1}{2} \mathcal{A}^{\prime \prime}(\hat{X}): \mathcal{P}+\mathcal{O}\left(\mathbb{E}\left(|X-\hat{X}|^{3}\right)\right)
$$

and the propagated covariance by

$$
\mathbb{E}\left((Y-\mathbb{E}(Y))\left(Y-\mathbb{E}(Y)^{T}\right)=\mathcal{A}^{\prime}(\hat{X}) \mathcal{P}\left(\mathcal{A}^{\prime}(\hat{X})\right)^{T}+\mathcal{O}\left(\mathbb{E}\left(|X-\hat{X}|^{3}\right)\right) .\right.
$$

We see in 22 that the simple propagation of the mean value by the nonlinear operator is only locally second order accurate (the term $\mathcal{A}^{\prime \prime}(\hat{X}): \mathcal{P}$ being second order in $|X-\hat{X}|)$. This will be improved with the technique presented in section 3.4

Another way to approximate means and covariances consists of choosing a set of vectors $X^{(i)}, 1 \leq i \leq r$, called particles, which are propagated with the operator $\mathcal{A}(\cdot)$, i.e.,

$$
Y^{(i)}=\mathcal{A}\left(X^{(i)}\right) \quad, 1 \leq i \leq r .
$$

Then, the mean and covariances are obtained by computing the empirical statistics of the propagated particles $Y^{(i)}$. Of course, to be effective, the particles have to be carefully chosen. In this work, we use this type of mean and covariance approximations in the context of the Unscented Kalman Filter [15, [16, which is based on deterministic particles. It will be briefly presented in the next section.

The UKF filter presents a lot of similarities with the Ensemble Kalman Filter [7 which also uses a moderate number of particles - even if this number is often a larger than for UKF - to compute empirical statistics. Moreover nonlinear particle filters consider a random generation of the particles $X^{(i)}$ according to a given probability density function. These filters usually involve a very large number of particles, which yields a very large number of solutions of the forward problem. We refer for example to [29].

\subsection{Unscented transforms and application to Kalman fil- tering}

The Unscented Kalman Filter (UKF) algorithm is based on the unscented transformation. Its principle is to approximate the nonlinear propagation of the mean and the covariance of a random vector via the propagation of well-chosen deterministic particles.

The basic idea can easily be explained in one dimension. Let $X$ be a random variable in $\mathbb{R}$ (so $N=1)$, let $\hat{X}$ denote its mean value $\mathbb{E}(X)$ and $\sigma=\sqrt{\operatorname{Var}(X)}$ its standard deviation. Two particles are defined: $\hat{X}^{(1)}=\hat{X}+\sigma, \hat{X}^{(2)}=\hat{X}-\sigma$. 
These particles are constructed by adding to the known state $\hat{X}$ two values $\sigma$ and $-\sigma$ called sigma-points. The empirical mean value of the particles, namely $\left(\hat{X}^{(1)}+\hat{X}^{(2)}\right) / 2$, is by construction equals to the mean value $\hat{X}$. In order to evaluate the empirical mean value of the particles propagated by the nonlinear operator, let us consider the Taylor expansions:

$$
\begin{aligned}
& \mathcal{A}\left(\hat{X}^{(1)}\right)=\mathcal{A}(\hat{X})+\sigma \mathcal{A}^{\prime}(\hat{X})+\frac{\sigma^{2}}{2} \mathcal{A}^{\prime \prime}(\hat{X})+\mathcal{O}\left(\left|\hat{X}^{(1)}-\hat{X}\right|^{3}\right), \\
& \mathcal{A}\left(\hat{X}^{(2)}\right)=\mathcal{A}(\hat{X})-\sigma \mathcal{A}^{\prime}(\hat{X})+\frac{\sigma^{2}}{2} \mathcal{A}^{\prime \prime}(\hat{X})+\mathcal{O}\left(\left|X^{(2)}-\hat{X}\right|^{3}\right) .
\end{aligned}
$$

The empirical mean is by construction

$\hat{Y} \stackrel{\text { def }}{=} \frac{1}{2}\left(\mathcal{A}\left(X^{(1)}\right)+\mathcal{A}\left(X^{(2)}\right)\right)=\mathcal{A}(\hat{X})+\frac{\sigma^{2}}{2} \mathcal{A}^{\prime \prime}(\hat{X})+\mathcal{O}\left(\left|\hat{X}^{(1)}-\hat{X}\right|^{3}\right)+\mathcal{O}\left(\left|\hat{X}^{(2)}-\hat{X}\right|^{3}\right)$.

Comparing $(22)$ and 24 , we see that the empirical mean of the propagated particle is an approximation of $\mathbb{E}(\mathcal{A}(X))$ better than $\mathcal{A}(\hat{X})$ since it includes by construction the second order term $\sigma^{2} \mathcal{A}^{\prime \prime}(\hat{X}) / 2$. Note that the covariance are approximated with the same order of accuracy in the two methods.

This idea can be generalized in $N$-dimensions by defining appropriate sigmapoints and their respective weights - see 23 for a comprehensive review. The natural generalization of the one-dimensional standard deviation is the square root of the covariance matrix. Its computation, for example by a Cholesky factorization, is the most expensive part of the UKF algorithm, but usually negligible compared to the propagation step for the problems considered in this work.

The empirical mean and covariance are defined by:

$\bar{X} \stackrel{\text { def }}{=} E_{\alpha}\left(X^{(*)}\right) \stackrel{\text { def }}{=} \sum_{1 \leq i \leq r} \alpha_{i} X^{(i)} \quad, \quad \operatorname{Cov}_{\alpha}\left(X^{(*)}, Z^{(*)}\right) \stackrel{\text { def }}{=} \sum_{1 \leq i \leq r} \alpha_{i}\left(X^{(i)}-\bar{X}\right)\left(Z^{(i)}-\bar{Z}\right)^{T}$,

where the weights $\alpha_{i} \in \mathbb{R}^{+}$are given and depend on the choice of the sigmapoints. In the present work, we use the ones proposed in [14, only based on $r=N+1$ particles (see Appendix A).

It remains to explain how to use the unscented transformation in the filtering algorithm. The idea is to replace the formulae (11) used in the original Kalman algorithm by the evaluations of the empirical covariance and mean value of the particles. More precisely, $P^{X Z}$ is replaced by $\operatorname{Cov}_{\alpha}\left(Y^{(*)}, Z^{(*)}\right)$, with $Y^{(i)}=$ $\mathcal{A}\left(X^{(i)}\right)$ and $Z^{(i)}=H\left(Y^{(i)}\right)$, and $P^{Z}$ is replaced by $W+\operatorname{Cov}_{\alpha}\left(Z^{(*)}, Z^{(*)}\right)$.

To summarize, here is the UKF algorithm. Assume that the sigma-points $I^{(i)}, 1 \leq i \leq r$, are given, as well as $\hat{X}_{0}^{+}$and $P_{0}^{+}$. For $n \geq 0$, we have a 3 steps recursive algorithm:

- Sampling. Generation of the particles:

$$
\begin{cases}C_{n}=\sqrt{P_{n}^{+}} & \text {(Cholesky factorization) } \\ \hat{X}_{n}^{(i)+}=\hat{X}_{n}^{+}+C_{n}^{T} I^{(i)}, & 1 \leq i \leq r\end{cases}
$$


- Prediction. Resolution of one time step of the model for each particle (can naturally be done in parallel) and computation of the empirical mean value and covariance:

$$
\left\{\begin{array}{l}
\hat{X}_{n+1}^{(i)-}=A_{n+1}\left(\hat{X}_{n}^{(i)+}\right) \quad, 1 \leq i \leq r \\
\hat{X}_{n+1}^{-}=E_{\alpha}\left(\hat{X}_{n+1}^{(*)}\right) \\
P_{n+1}^{-}=\operatorname{Cov}_{\alpha}\left(\hat{X}_{n+1}^{(*)-}, \hat{X}_{n+1}^{(*)-}\right)
\end{array}\right.
$$

- Correction.

$$
\left\{\begin{array}{l}
Z_{n+1}^{(i)}=H\left(\hat{X}_{n+1}^{(i)-}\right) \quad, 1 \leq i \leq r \\
P_{n+1}^{Z}=\operatorname{Cov}_{\alpha}\left(Z_{n+1}^{(*)}, Z_{n+1}^{(*)}\right)+W_{n+1} \\
P_{n+1}^{X Z}=\operatorname{Cov}_{\alpha}\left(\hat{X}_{n+1}^{(*)-}, \hat{Z}_{n+1}^{(*)}\right) \\
K_{n+1}=P_{n+1}^{X Z}\left(P_{n+1}^{Z}\right)^{-1} \\
\hat{X}_{n+1}^{+}=\hat{X}_{n+1}^{-}+K_{n+1}\left(Z_{n+1}-E_{\alpha}\left(Z_{n+1}^{(*)}\right)\right) \\
P_{n+1}^{+}=P_{n+1}^{-}-P_{n+1}^{X Z}\left(P_{n+1}^{Z}\right)^{-1}\left(P_{n+1}^{X Z}\right)^{T}
\end{array}\right.
$$

\subsection{Factorized formulation of the UKF}

In this section, we show how the construction of $P_{n+1}^{+}, P_{n+1}^{X Z}$ and $P_{n+1}^{Z}$ is modified when the covariance matrix $P_{n+1}^{-}$can be factorized in the form $L U^{-1} L^{T}$, with $L \in \mathcal{M}_{N, p}$ and $U \in \mathcal{M}_{p, p}$. This will be useful to derive the reduced-order UKF algorithm in the next section.

We consider a family of sigma-points $I^{(i)} \in \mathbb{R}^{p}, i=1 \ldots r$. It is proved in [23. Sect. 2.2.1] that the empirical covariance of the particles $X^{(i)}$ and $Z^{(i)}$ (see (25)) can be expressed as

$$
\operatorname{Cov}_{\alpha}\left(X^{(*)}, Z^{(*)}\right)=\left[X^{(*)}\right] D_{\alpha}\left[I^{(*)}\right]^{T}\left(\left[I^{(*)}\right] D_{\alpha}\left[I^{(*)}\right]^{T}\right)^{-1}\left[I^{(*)}\right] D_{\alpha}\left[Z^{(*)}\right]^{T}
$$

where $\left[X^{(*)}\right] \in \mathcal{M}_{N, r}$ is the matrix whose column $i$ corresponds to the particle $X^{(i)}, 1 \leq i \leq r$, and where $D_{\alpha} \stackrel{\text { def }}{=} \operatorname{diag}\left[\alpha_{i}\right] \in \mathcal{M}_{r, r}$ is the diagonal matrix containing the weights of formulae 25 .

The covariance matrix $P_{n+1}^{-}$is by definition $\operatorname{Cov}_{\alpha}\left(\hat{X}_{n+1}^{(*)-}, \hat{X}_{n+1}^{(*)-}\right)$. Thus, according to 29 , we rewrite it as

$$
P_{n+1}^{-}=L_{n+1}^{X} P_{\alpha}^{-1}\left(L_{n+1}^{X}\right)^{T},
$$

with

$$
L_{n+1}^{X} \stackrel{\text { def }}{=}\left[\hat{X}_{n+1}^{(*)-}\right] D_{\alpha}\left[I^{(*)}\right]^{T} \text { and } P_{\alpha} \stackrel{\text { def }}{=}\left[I^{(*)}\right] D_{\alpha}\left[I^{(*)}\right]^{T} .
$$

Similarly, applying again $29 p$ to $P_{n+1}^{Z}$ and $P_{n+1}^{X Z}$ (see their definition in (28)):

$$
P_{n+1}^{Z}=W_{n+1}+L_{n+1}^{Z} P_{\alpha}^{-1}\left(L_{n+1}^{Z}\right)^{T}, P_{n+1}^{X Z}=L_{n+1}^{X} P_{\alpha}^{-1}\left(L_{n+1}^{Z}\right)^{T},
$$

with

$$
L_{n+1}^{Z} \stackrel{\text { def }}{=}\left[Z_{n+1}^{(*)}\right] D_{\alpha}\left[I^{(*)}\right]^{T} .
$$

Thus, the a posteriori covariance $P_{n+1}^{+}$can be written as:

$$
\begin{aligned}
P_{n+1}^{+} & =P_{n+1}^{-}-P_{n+1}^{X Z}\left(P_{n+1}^{Z}\right)^{-1}\left(P_{n+1}^{X Z}\right)^{T} \\
& =L_{n+1}^{X}\left(P_{\alpha}^{-1}-P_{\alpha}^{-1}\left(L_{n+1}^{Z}\right)^{T}\left(W_{n+1}+L_{n+1}^{Z} P_{\alpha}^{-1}\left(L_{n+1}^{Z}\right)^{T}\right)^{-1} L_{n+1}^{Z} P_{\alpha}^{-1}\right)\left(L_{n+1}^{X}\right)^{T} \\
& =L_{n+1}^{X} U_{n+1}^{-1}\left(L_{n+1}^{X}\right)^{T}
\end{aligned}
$$


where $U_{n+1}$ is defined, applying the Woodbury inversion lemma, by

$$
U_{n+1} \stackrel{\text { def }}{=} P_{\alpha}+\left(L_{n+1}^{Z}\right)^{T} W_{n+1}^{-1} L_{n+1}^{Z} \text {. }
$$

Next, applying again the Woodbury inversion lemma to $P_{n+1}^{Z}$, we obtain:

$$
\left(P_{n+1}^{Z}\right)^{-1}=W_{n+1}^{-1}-W_{n+1}^{-1} L_{n+1}^{Z} U_{n+1}^{-1}\left(L_{n+1}^{Z}\right)^{T} W_{n+1}^{-1} .
$$

Thus, we deduce the factorized form of the Kalman-like matrix $K_{n+1}=P_{n+1}^{X Z}\left(P_{n+1}^{Z}\right)^{-1}$ :

$$
\begin{aligned}
K_{n+1} & =\left(L_{n+1}^{X} P_{\alpha}^{-1}\left(L_{n+1}^{Z}\right)^{T}\right)\left(W_{n+1}^{-1}-W_{n+1}^{-1} L_{n+1}^{Z} U_{n+1}^{-1}\left(L_{n+1}^{Z}\right)^{T} W_{n+1}^{-1}\right) \\
& =L_{n+1}^{X} U_{n+1}^{-1}\left(L_{n+1}^{Z}\right)^{T} W_{n+1}^{-1} .
\end{aligned}
$$

The key point here is to be able to perform operations with $P_{n+1}^{+}$and $K_{n+1}$ using only $L_{n+1}^{X}, L_{n+1}^{Z}$ and $P_{\alpha}$, and not directly $P_{n+1}^{-}$. This property proves to be extremely useful when the rank of $P_{n+1}^{-}$is much smaller than the size of $X$, as will be shown in the next section.

\section{REMARK 1}

According to (9), $K=P^{+} H^{T} W^{-1}$. Thus, using the expression (34) of $P^{+}$, we obtain $K=L^{X} U^{-1}\left(L^{X}\right)^{T} H^{T} W^{-1}$. By comparison with (37), we see that $L^{Z}$ formally corresponds to $H L^{X}$, the "observed state sensitivity". Matrix $L^{X}\left(L^{\theta}\right)^{-1}$ corresponds to the sensitivity of the state $X$ with respect to the parameters $\theta$. The factorization formula $(29)$ is instrumental in identifying these analogies. We refer to 23 for more details.

\subsection{Reduced-order UKF for parameter estimation}

State and parameter estimation In principle, the UKF algorithm can easily be generalized to those cases when the uncertainties also affect the parameters. To do so, we consider an augmented state $\mathcal{X}=(X, \theta)$ whose size is $\mathcal{N}=N+p$. At $t=0, \mathcal{X}(0)=\mathcal{X}_{0}+\zeta^{\mathcal{X}}=\left(X_{0}, \theta_{0}\right)+\left(\zeta^{X}, \zeta^{\theta}\right)$. The extended dynamical system reads:

$$
\mathcal{X}_{n+1} \stackrel{\text { def }}{=}\left(X_{n+1}, \theta_{n+1}\right)=\mathcal{A}_{n+1}\left(\mathcal{X}_{n}\right),
$$

with the initial condition $\mathcal{X}_{0}=\left(X_{0}, \theta_{0}\right)$. When the parameters $\theta$ are constant in time, $\mathcal{A}_{n+1}$ is simply defined by $\mathcal{A}_{n+1}\left(\mathcal{X}_{n}\right)=\left(A_{n+1}\left(X_{n}, \theta_{n}\right), \theta_{n}\right)$. Then, even with a small number of parameters, classical Kalman-like filter cannot be applied to this augmented state because of the initial state size. However, if we assume that the uncertainties only affect the part $\theta$ of vector $\mathcal{X}$, we will show in the next paragraph how to use a reduced order filter formulation which limits the computation of the filter operator to a subspace of small dimension - here the parameter space - and therefore can be computed.

Reduced-order UKF We now have all the material to present the reducedorder UKF algorithm that is used in this paper to estimate some parameters of a fluid-structure interaction system.

As explained above, any Kalman-like algorithm can be used to estimate the state and the parameter by simply considering an augmented dynamical system 
in the variable $\mathcal{X}=(X, \theta)$. Nevertheless, as already mentioned, this approach is intractable with large system as those considered here. To circumvent this difficulty, the choice made in this study is to assume that the uncertainty is limited to the $p$ parameters $\theta$. Thus, the covariance matrix has a rank $p$ and we can take advantage from the factorized formulation of Section 3.5 .

Suppose that we do not have any uncertainties in the initial condition of the state, i.e., $\zeta^{X}=0$. Then the initial covariance can be factorized:

$$
P_{0}^{+}=\left[\begin{array}{cc}
0 & 0 \\
0 & \operatorname{Cov}\left(\zeta^{\theta}\right)
\end{array}\right]=\left[\begin{array}{c}
L_{0}^{X} \\
L_{0}^{\theta}
\end{array}\right] U_{0}^{-1}\left[\begin{array}{ll}
\left(L_{0}^{X}\right)^{T} & \left.\left(L_{0}^{\theta}\right)^{T}\right],
\end{array}\right.
$$

where $U_{0} \stackrel{\text { def }}{=}\left(\operatorname{Cov}\left(\zeta^{\theta}\right)\right)^{-1}$ is a (small) $p \times p$ matrix, $L_{0}^{x} \stackrel{\text { def }}{=} 0, L_{0}^{\theta} \stackrel{\text { def }}{=} \mathbb{1}$. Hence, and this is a key point, we only need $p+1$ sigma-points in $\mathbb{R}^{p}$ for the sampling step in the UKF algorithm:

$$
\left\{\begin{array}{l}
C_{0}=\sqrt{U_{0}^{-1}} \quad \text { (Cholesky factorization) } \\
\hat{X}_{0}^{(i)+}=\hat{X}_{0}^{+}+L_{0}^{X} C_{0}^{T} I^{(i)}=\hat{X}_{0}^{+}, \quad 1 \leq i \leq p+1 \\
\hat{\theta}_{0}^{(i)+}=\hat{\theta}_{0}^{+}+L_{0}^{\theta} C_{0}^{T} I^{(i)}, \quad 1 \leq i \leq p+1
\end{array}\right.
$$

Reduced order estimation algorithm Consider the simplex sigma-points $I^{(i)}, 1 \leq i \leq p+1$, their weights collected in the matrix $D_{\alpha} \stackrel{\text { def }}{=} \operatorname{diag}\left[\alpha_{i}\right] \in$ $\mathcal{M}_{p+1, p+1}$. Then, for given values of $\hat{X}_{0}, \hat{\theta}_{0}$ and $\operatorname{Cov}\left(\zeta^{\theta}\right)$, perform

- Initialization: initialize the sensitivities as

$$
L_{0}^{\theta}=\mathbb{1}, \quad L_{0}^{X}=0, \quad U_{0}=\operatorname{Cov}\left(\zeta_{\theta}\right)^{-1}
$$

- Sampling: generate the particles by

$$
\begin{cases}C_{n}=\sqrt{U_{n}^{-1}} & \text { (Cholesky factorization) } \\ \hat{X}_{n}^{(i)+}=\hat{X}_{n}^{+}+L_{n}^{X} C_{n}^{T} I^{(i)}, \quad 1 \leq i \leq p+1 \\ \hat{\theta}_{n}^{(i)+}=\hat{\theta}_{n}^{+}+L_{n}^{\theta} C_{n}^{T} I^{(i)}, \quad 1 \leq i \leq p+1\end{cases}
$$

- Prediction: propagate the particles with the dynamical system

$$
\left\{\begin{array}{l}
\left(\hat{X}_{n+1}^{(i)-}, \hat{\theta}_{n+1}^{(i)-}\right)=A_{n+1}\left(\hat{X}_{n}^{(i)+}, \hat{\theta}_{n}^{(i)+}\right) \\
\hat{X}_{n+1}^{-}=E_{\alpha}\left(\hat{X}_{n+1}^{(*)-}\right) \\
\hat{\theta}_{n+1}^{-}=E_{\alpha}\left(\hat{\theta}_{n+1}^{(*)-}\right)
\end{array}\right.
$$

- Correction: use the innovation $\Gamma_{n+1}^{(i)}=Z_{n+1}-H\left(\hat{X}_{n+1}^{(i)-}\right)(1 \leq i \leq p+1)$ to correct the predicted state and parameters

$$
\left\{\begin{array}{l}
L_{n+1}^{X}=\left[\hat{X}_{n+1}^{(*)-}\right] D_{\alpha}\left[I^{(*)}\right]^{T} \in \mathcal{M}_{N, p} \\
L_{n+1}^{\theta}=\left[\hat{\theta}_{n+1}^{(*)-}\right] D_{\alpha}\left[I^{(*)}\right]^{T} \in \mathcal{M}_{p} \\
L_{n+1}^{Z}=\left[\Gamma_{n+1}^{(*)}\right] D_{\alpha}\left[I^{(*)}\right]^{T} \\
U_{n+1}=P_{\alpha}+\left(L_{n+1}^{Z}\right)^{T} W_{n+1}^{-1} L_{n+1}^{Z} \in \mathcal{M}_{p} \\
\hat{X}_{n+1}^{+}=\hat{X}_{n+1}^{-}+L_{n+1}^{X} U_{n+1}^{-1}\left(L_{n+1}^{Z}\right)^{T} W_{n+1}^{-1} E_{\alpha}\left(\Gamma_{n+1}^{(*)}\right) \\
\hat{\theta}_{n+1}^{+}=\hat{\theta}_{n+1}^{-}+L_{n+1}^{\theta} U_{n+1}^{-1}\left(L_{n+1}^{Z}\right)^{T} W_{n+1}^{-1} E_{\alpha}\left(\Gamma_{n+1}^{(*)}\right)
\end{array}\right.
$$




\section{REMARK 2}

We have implicitly used some specific properties of the simplex sigma-points in the derivation of the algorithm as fully described in [23. For more general sigma-points, the algorithm is more intricate. We refer to [23] and in particular its erratum 22 .

Of course in practice, the state $X$ also contains uncertainties. Although this problem will not be addressed in this paper, let us just mention that a possible solution consists of using the reduced order UKF in combination with another filtering technique, like a Luenberger observer [20. This approach consists of adding a feedback term in the time-continous dynamics

$$
\dot{\hat{X}}=A(\hat{X}, \theta)+K_{L}(Z-H(\hat{X})),
$$

where $K_{L}$ has to be designed for each physics and each type of observations in order to make the estimation error $X-\hat{X}$ decrease rapidly. We refer the reader to 24] where this approach has been proposed and applied to solid mechanics. For a study about Luenberger observers in fluid-structure problems, we refer to 3 .

\section{Reduced Order filtering for FSI problems}

\subsection{Fluid-Structure algorithm}

Many procedures are known to couple the fluid (1) and the solid (2) problems (see, e.g., [8, 9] and the references therein). In this work, we choose a semi-implicit partitioned algorithm. The scheme is called "partitioned" because it keeps independent the fluid and structure solvers and it is called "semiimplicit" because only a part of the fluid problem needs subiterations with the structure. A Newmark scheme is used in the solid and a first order ChorinTemam projection method in the fluid. The coupling scheme treats explicitly the ALE-advection-diffusion step and implicitly the projection step, following the approach proposed in [10, 11.

Considering the variables $\left(\tilde{\boldsymbol{u}}^{n}, \widehat{\boldsymbol{y}}_{\mathrm{f}}^{n}, \widehat{\boldsymbol{y}}^{n}, \widehat{\boldsymbol{w}}^{n}, \widehat{\boldsymbol{a}}^{n}\right)$ which correspond to the fullydiscrete fluid velocity field, fluid domain displacement, solid displacement, solid velocity and solid acceleration, respectively, the complete fluid-structure algorithm reads: Given the initial condition $\left(\tilde{\boldsymbol{u}}^{0}, \widehat{\boldsymbol{y}}_{\mathrm{f}}^{0}, \widehat{\boldsymbol{y}}^{0}, \widehat{\boldsymbol{w}}^{0}, \widehat{\boldsymbol{a}}^{0}\right)$, find $\left(\tilde{\boldsymbol{u}}^{n}, \widehat{\boldsymbol{y}}_{\mathrm{f}}^{n}, \widehat{\boldsymbol{y}}^{n}, \widehat{\boldsymbol{w}}^{n}, \widehat{\boldsymbol{a}}^{n}\right)$, for $n>0$, as follows:

1. For $n=0$ initialize the pressure as:

$$
\begin{array}{rcc}
-\Delta p^{0}=-\frac{\rho_{\mathrm{f}}}{\tau} \boldsymbol{\nabla} \cdot \tilde{\boldsymbol{u}}^{0} & \text { in } & \Omega_{\mathrm{f}}^{0}, \\
\boldsymbol{\nabla} p^{0} \cdot \boldsymbol{n}=0 & \text { on } & \Gamma^{\text {in }}, \\
p^{0}=\bar{p}\left(t_{0}\right) & \text { on } & \Gamma^{\text {out }}, \\
\nabla p^{0} \cdot \boldsymbol{n}=-\frac{\rho_{\mathrm{f}}}{\tau}\left(\widehat{\boldsymbol{w}}^{-\frac{1}{2}}-\tilde{\boldsymbol{u}}^{0}\right) \cdot \boldsymbol{n} & \text { on } & \Sigma^{0},
\end{array}
$$

with $\widehat{\boldsymbol{w}}^{-\frac{1}{2}}=\widehat{\boldsymbol{w}}^{0}-\tau \widehat{\boldsymbol{a}}^{0} / 2$ and $\Omega_{\mathrm{f}}^{0}=\widehat{\Omega}_{\mathrm{f}}$.

2. ALE-Advection-diffusion step (explicit coupling with the solid): 
- Update fluid domain:

$$
\begin{gathered}
\widehat{\boldsymbol{y}}_{\mathrm{f}}^{n+1}=\operatorname{Ext}\left(\left.\widehat{\boldsymbol{y}}^{n}\right|_{\widehat{\Sigma}}\right), \quad \Omega_{\mathrm{f}}^{n+1}=\left(\boldsymbol{I}_{\widehat{\Omega}_{\mathrm{f}}}+\widehat{\boldsymbol{y}}_{\mathrm{f}}^{n+1}\right)\left(\widehat{\Omega}_{\mathrm{f}}\right), \\
\boldsymbol{w}_{\mathrm{f}}^{n+1}=\partial_{\tau} \widehat{\boldsymbol{y}}_{\mathrm{f}}^{n+1} \quad \text { in } \quad \Omega_{\mathrm{f}}^{n+1} .
\end{gathered}
$$

- Fluid viscous sub-step:

$$
\left\{\begin{array}{rcc}
\left.\rho_{\mathrm{f}} \frac{\tilde{\boldsymbol{u}}^{n+1}-\tilde{\boldsymbol{u}}^{n}}{\tau}\right|_{\widehat{\boldsymbol{x}}}+\rho_{\mathrm{f}}\left(\tilde{\boldsymbol{u}}^{n}-\boldsymbol{w}_{\mathrm{f}}^{n+1}\right) \cdot \boldsymbol{\nabla} \tilde{\boldsymbol{u}}^{n+1}-2 \mu \boldsymbol{\nabla} \cdot \boldsymbol{\epsilon}\left(\tilde{\boldsymbol{u}}^{n+1}\right)=-\boldsymbol{\nabla} p^{n} & \text { in } \quad \Omega_{\mathrm{f}}^{n+1}, \\
\tilde{\boldsymbol{u}}^{n+1}=\boldsymbol{u}_{\mathrm{in}}\left(t_{n+1}\right) & \text { on } \quad \Gamma^{\text {in }}, \\
2 \mu \boldsymbol{\epsilon}\left(\tilde{\boldsymbol{u}}^{n+1}\right) \boldsymbol{n}_{\mathrm{f}}=\mathbf{0} & \text { on } \quad \Gamma^{\mathrm{out}}, \\
\tilde{\boldsymbol{u}}^{n+1}=\widehat{\boldsymbol{w}}^{n-\frac{1}{2}} & \text { on } & \Sigma^{n+1} .
\end{array}\right.
$$

3. Projection step (implicit coupling with the solid): Initialize $\widehat{\boldsymbol{w}}^{n+\frac{1}{2}}=$ $\widehat{\boldsymbol{w}}^{n-\frac{1}{2}}$, and iterate $(k>0)$ until

$$
\sqrt{\left|\widehat{\boldsymbol{y}}_{k}^{n+1}-\widehat{\boldsymbol{y}}_{k-1}^{n+1}\right|^{2}+\left|\widehat{\boldsymbol{w}}_{k}^{n+\frac{1}{2}}-\widehat{\boldsymbol{w}}_{k-1}^{n+\frac{1}{2}}\right|^{2}}<\varepsilon \quad, \quad \text { on } \Sigma^{n+1} .
$$

with $\varepsilon$ a given tolerance.

- Fluid projection sub-step:

$$
\left\{\begin{array}{rll}
-\Delta p_{k}^{n+1}=-\frac{\rho_{\mathrm{f}}}{\tau} \boldsymbol{\nabla} \cdot \tilde{\boldsymbol{u}}^{n+1} & \text { in } & \Omega_{\mathrm{f}}^{n+1}, \\
\boldsymbol{\nabla} p_{k}^{n+1} \cdot \boldsymbol{n}=0 & \text { on } & \Gamma^{\text {in }}, \\
p_{k}^{n+1}=\bar{p}\left(t_{n+1}\right) & \text { on } & \Gamma^{\text {out }} \\
\nabla p_{k}^{n+1} \cdot \boldsymbol{n}=-\frac{\rho_{\mathrm{f}}}{\tau}\left(\widehat{\boldsymbol{w}}_{k-1}^{n+\frac{1}{2}}-\tilde{\boldsymbol{u}}^{n+1}\right) \cdot \boldsymbol{n} & \text { on } & \Sigma^{n+1} .
\end{array}\right.
$$

- Solid:

$$
\left\{\begin{array}{rcc}
\rho_{\mathrm{s}} \partial_{\tau} \widehat{\boldsymbol{w}}_{k}^{n+1}-\boldsymbol{\nabla} \cdot \boldsymbol{\sigma}_{\mathrm{s}}\left(\widehat{\boldsymbol{y}}_{k}^{n+\frac{1}{2}}\right)=\mathbf{0} & \text { in } & \widehat{\Omega}_{\mathrm{s}}, \\
\partial_{\tau} \widehat{\boldsymbol{y}}_{k}^{n+1}=\widehat{\boldsymbol{w}}_{k}^{n+\frac{1}{2}} & \text { in } & \widehat{\Omega}_{\mathrm{s}}, \\
\widehat{\boldsymbol{y}}_{k}^{n+1}=\mathbf{0} & \text { on } & \Gamma^{\mathrm{d}}, \\
\boldsymbol{\sigma}_{\mathrm{s}}\left(\widehat{\boldsymbol{y}}_{k}^{n+\frac{1}{2}}\right) \boldsymbol{n}_{\mathrm{s}}=\mathbf{0} & \text { on } & \Gamma^{\mathrm{n}}, \\
\boldsymbol{\sigma}_{\mathrm{s}}\left(\widehat{\boldsymbol{y}}_{k}^{n+\frac{1}{2}}\right) \boldsymbol{n}_{\mathrm{s}}=-J_{\mathrm{f}}^{n+1} \widehat{\boldsymbol{\sigma}}_{\mathrm{f}}\left(\tilde{\boldsymbol{u}}^{n+1}, p_{k}^{n+1}\right)\left(\boldsymbol{F}_{\mathrm{f}}^{n+1}\right)^{-\mathrm{T}} \boldsymbol{n} & \text { on } & \widehat{\Sigma} .
\end{array}\right.
$$

4. Set

$$
\widehat{\boldsymbol{y}}^{n+1}=\widehat{\boldsymbol{y}}_{k}^{n+1}, \quad \widehat{\boldsymbol{w}}^{n+1}=\widehat{\boldsymbol{w}}_{k}^{n+1}, \quad \widehat{\boldsymbol{a}}^{n+1}=\frac{\widehat{\boldsymbol{w}}^{n+1}-\widehat{\boldsymbol{w}}^{n}}{\tau}, \quad n=n+1
$$

and go to Step 1.

In general, a matrix free Newton method is used to solve the interface equation resulting from the coupling between (43) and (44). In this work, since only linear solids are considered, the interface problem is simply solved with a matrix free GMRES algorithm.

The fluid and the solid subproblems are dicretized in space with the $\mathbb{P}_{1}$ finite element. The advection in the fluid is stabilized with a streamline diffusion term. 


\subsection{Algorithmic aspects of FSI filtering}

Implementation Beside its efficiency, a very appealing feature of the proposed approach is the simplicity of its implementation. In algorithm (39a)$(39 \mathrm{~d})$, steps $39 \mathrm{a}),(39 \mathrm{~b})$ and $(39 \mathrm{~d})$ are totally independent of the problem and can be implemented in an external software. Since this part is generic, it is very simple to change the number and the kind of parameters that are used in the filter. Step (39c) corresponds to one time step of the dynamical system with a given initial condition. It therefore only requires to be able to "restart" the solvers from any state.

Particle initialization Let us give some more details about the "restart" of the fluid-structure algorithm, since this is an important aspect of the estimation procedure.

In our case, the discrete state $X_{n}$ corresponds to the degrees of freedom of $\left(\tilde{\boldsymbol{u}}^{n}, \widehat{\boldsymbol{y}}_{\mathrm{f}}^{n}, \widehat{\boldsymbol{y}}^{n-\frac{1}{2}}, \widehat{\boldsymbol{w}}^{n-\frac{1}{2}}, \widehat{\boldsymbol{a}}^{n}\right)$. At each time step, and for each particle separately, the following steps are performed:

- In the solid:

- Recover $\widehat{\boldsymbol{y}}^{n}$ and $\widehat{\boldsymbol{w}}^{n}$ by

$$
\widehat{\boldsymbol{y}}^{n}=\widehat{\boldsymbol{y}}^{n-\frac{1}{2}}+\frac{\tau}{2} \widehat{\boldsymbol{w}}^{n-\frac{1}{2}}, \widehat{\boldsymbol{w}}^{n}=\widehat{\boldsymbol{w}}^{n-\frac{1}{2}}+\frac{\tau}{2} \widehat{\boldsymbol{a}}^{n} .
$$

- In the fluid:

- Reconstruct $\Omega_{\mathrm{f}}^{n}=\left(\boldsymbol{I}_{\widehat{\Omega}_{\mathrm{f}}}+\widehat{\boldsymbol{y}}_{\mathrm{f}}^{n}\right)\left(\widehat{\Omega}_{\mathrm{f}}\right)$

- Reconstruct the pressure $p^{n}$ by solving

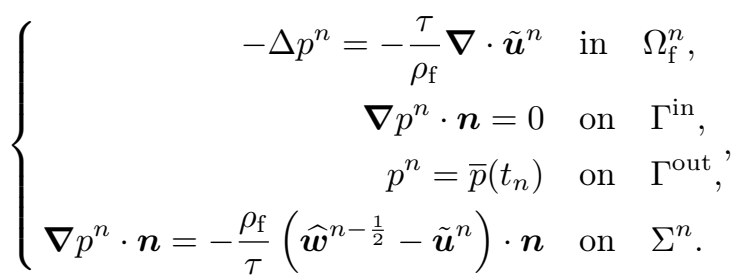

Then perform steps 2 and 3 of the semi-implicit algorithm to get the new state by $X_{n+1}=\left(\tilde{\boldsymbol{u}}^{n+1}, \widehat{\boldsymbol{y}}_{\mathrm{f}}^{n+1}, \widehat{\boldsymbol{y}}^{n+\frac{1}{2}}, \widehat{\boldsymbol{w}}^{n+\frac{1}{2}}, \widehat{\boldsymbol{a}}^{n+1}\right)$.

Total vs. incremental ALE In many ALE solvers, the domain is updated in an incremental way, from $\Omega^{n}$ to $\Omega^{n+1}$, i.e.,

$$
\widehat{\boldsymbol{w}}_{\mathrm{f}}^{n+1}=\operatorname{Ext}\left(\left.\widehat{\boldsymbol{w}}^{n-\frac{1}{2}}\right|_{\Sigma^{n}}\right), \quad \Omega_{\mathrm{f}}^{n+1}=\Omega_{\mathrm{f}}^{n}+\tau \widehat{\boldsymbol{w}}_{\mathrm{f}}^{n} .
$$

This approach is not suitable in the present framework since it would results in incorrect fluid domains in each particle. This is the reason why we update the domain from $\widehat{\Omega}_{\mathrm{f}}$ using the Lagrangian displacement $\widehat{\boldsymbol{y}}_{\mathrm{f}}^{n}$. For very large displacements, this may complicate the computation of the mesh deformation (using for example a nonlinear pseudo-elasticity problem). In the applications presented in this work, a simple linear harmonic extension proved to be sufficient. 
Windkessel boundary conditions In blood flow simulations, the pressure $\bar{p}$ on $\Gamma^{\text {out }}$ is given by the ordinary differential equation (4). Its discrete form reads (assuming $P_{V}=0$ ):

$$
\left\{\begin{aligned}
\pi^{n+1} & =\frac{R_{d} \tau}{R_{d} C+\tau} \pi^{n}+\frac{R_{d} C}{R_{d} C+\tau} \tilde{Q}^{n}, \\
\bar{p}\left(t_{n+1}\right) & =R_{p} \tilde{Q}^{n+1}+\pi^{n+1}, \\
\tilde{Q}^{n} & =\int_{\Gamma_{\text {out }}} \tilde{\boldsymbol{u}}^{n} \cdot \boldsymbol{n},
\end{aligned}\right.
$$

where $R_{p}, R_{d}$ and $C$ are given constants. As already mentioned, when this kind of models is used for the fluid boundary conditions, $\pi^{n}$ has to be included in the state, i.e., $X_{n}=\left(\tilde{\boldsymbol{u}}^{n}, \widehat{\boldsymbol{y}}_{\mathrm{f}}^{n}, \pi^{n}, \widehat{\boldsymbol{y}}^{n-\frac{1}{2}}, \widehat{\boldsymbol{w}}^{n-\frac{1}{2}}, \widehat{\boldsymbol{a}}^{n}\right)$. When the system is "restarted", equation 46$)_{2}$ is used to define the value $\bar{p}\left(t_{n}\right)$ in 45$)_{3}$.

Parameter range constraints For physical reason, the parameters are usually restricted to a subset of $\mathbb{R}^{p}$. For instance, Young's modulus, densities and viscosities have to be positive. This constraint can simply be enforced by reparametrizing the physical parameters in such a way that the estimation can indeed be done in the whole space $\mathbb{R}^{p}$. In this work, the physical parameters (e.g. the Young's modulus) are written as $2^{\theta_{i}}$, and the estimation is performed on $\theta_{i}$. Note that this reparametrization modifies the statistical meaning of the covariance. For example, when the covariance on $\theta$ is equal to identity, the Young's modulus $2^{\theta}$ has the same probability to be twice or half as the initial value.

Choice of the covariance $W_{n}$ Due to the analogy with variational methods (i.e., comparing for instance (20) and (6)), we use $W_{n}^{-1}=\gamma M_{\Gamma_{\mathrm{m}}}$, with $M_{\Gamma_{\mathrm{m}}}$ the $L^{2}$ mass matrix of the observation region $\Gamma_{\mathrm{m}}$, or its diagonal-lumped version. Hence, we propose to choose

$$
\gamma=\beta w^{-1}
$$

where $\beta$ is a positive scalar value that has to be set and

$$
w^{-1}=\frac{\tau_{\text {obs }} \sigma^{-2}}{T_{\text {ref }} H_{\text {ref }}},
$$

where $T_{r e f}$ is a fixed reference time, $H_{r e f} \approx\left|\Gamma_{\mathrm{m}}\right|$, and $\tau_{o b s}$ is the time sampling of the observations, and assuming that $\zeta^{Z} \sim \mathcal{N}\left(0, \sigma^{2} I\right)$. These definitions is motivated by keeping dimensionless the tuning parameters associated with the measures and the a priori knowledge of the parameters. Note that the measure term "tends" to the continuous space-time norm when spatial and temporal resolution get higher.

\section{$5 \quad$ Numerical examples}

\subsection{Forward model description}

Consider the idealized abdominal aortic aneurysm (AAA) shown in Figure 2 . Its length is $22.95 \mathrm{~cm}$, its minimal diameter $1.7 \mathrm{~cm}$ (tubular part), its maximal 
diameter $4.98 \mathrm{~cm}$ (aneurysm) and its wall thickness $0.2 \mathrm{~cm}$. The solid volume is divided into 5 zones (along the axis), associated with different Young's moduli: $2^{\theta_{i}} M P a$, with $\theta_{1}=1.5, \theta_{2}=0.5, \theta_{3}=-1, \theta_{4}=0.2, \theta_{5}=1$. Its Poisson ratio is 0.46 , its density $1.2 \mathrm{~g} / \mathrm{cm}^{3}$ and viscosity $\eta_{\mathrm{s}}=10^{-3} \mathrm{~s}$. The fluid viscosity is $0.035 P o$ and its density $1 \mathrm{~g} / \mathrm{cm}^{3}$.

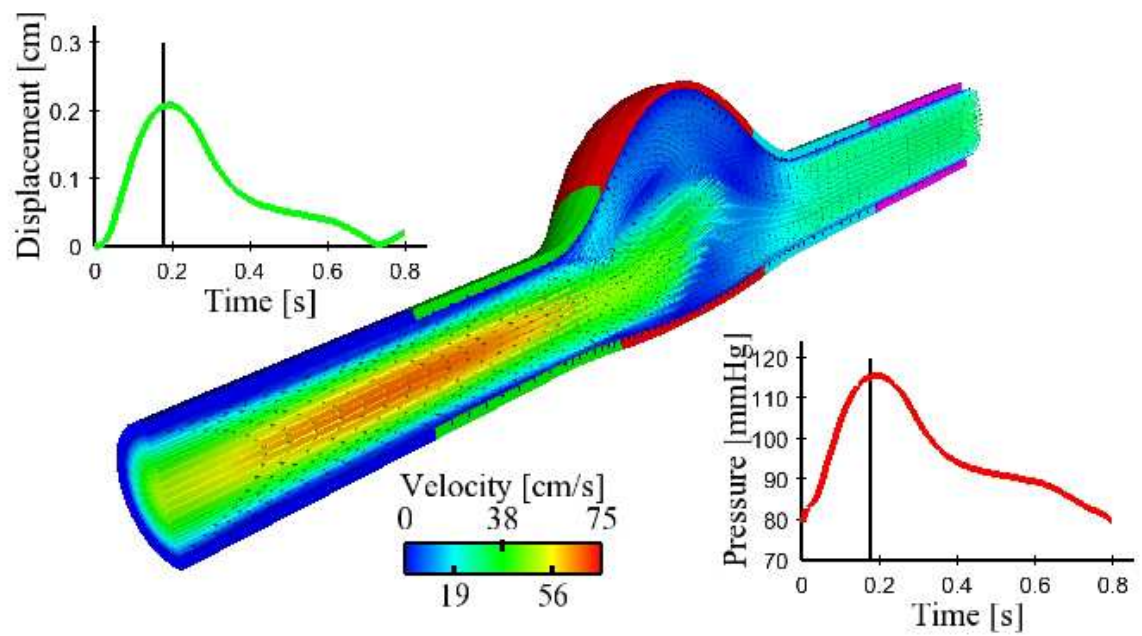

Figure 2: Idealized AAA: solid subregions (blue, green, red, cyan and magenta, respectively), fluid velocity field, pressure at the outlet and displacements at the aneurysm.

The boundary conditions are set as follows. The inlet and the outlet parts of the solid are clamped. In the fluid, a parabolic velocity profile is enforced at the inlet, with a realistic inflow (peak velocity $\approx 96 \mathrm{~cm} / \mathrm{s}$ ). At the fluid outlet, the three-dimensional model is coupled to a three-element Windkessel model (4) with $R_{p}=700, R_{d}=5 \cdot 10^{3}$ and $C=2 \cdot 10^{-4}$ (all three in CGS unit system) and $P_{V}=0$. The time step is $\tau=0.002 \mathrm{~s}$.

At $t=0$, the pressure is constant and equal to $80 \mathrm{mmHg}$, whereas all the other state variables are zero. During the whole simulation, the stress received by the structure is corrected by the initial one. Doing so, the solid only "feels" the difference with the diastolic phase. This is a simple way to account for the prestress in linear elastodynamics. We refer to [26], and references therein, for a discussion about prestress computation in a more general framework.

\subsection{Estimation of the Young's modulus}

Estimation setting We apply the reduced order UKF to estimate the Young's moduli, $E_{i}=2^{\hat{\theta}_{i}} M P a$, starting from $\hat{\theta}_{i}=0, i=1, \ldots, 5$. The measurements are the displacements on all nodes of the fluid-structure interface $\widehat{\Sigma}$. In other words, the observation operator $H$ consists of a $m \times n_{y}$ matrix (whose entries are only 0 or 1 ), where $n_{y}$ is the number of displacement degrees of freedom of 
the whole solid and $m$ is the number of displacement degrees of freedom at the interface $\widehat{\Sigma}$.

The initial parameter covariance is assumed to be

$$
\operatorname{Cov}\left(\zeta_{\theta}\right)=\alpha \mathbb{1}
$$

where $\alpha$ is a given positive parameter. The noised signal is given by

$$
Z_{n+1}=H Y_{n+1 / 2}^{d}+\sigma \hat{\zeta}
$$

where $Y_{n+1 / 2}^{d}$ is a synthetic solid displacement, $\hat{\zeta} \sim \mathcal{N}(0, I)$, and $\sigma=0.2 \mathrm{~mm}$, i.e., $10 \%$ of the maximal displacement at the solid wall.The measurements covariance is computed as indicated in Section 4.2. The scalar gain $w^{-1}$ is obtained by formulae 48 setting $T_{\text {ref }}=0.8 \mathrm{~s}, H_{\text {ref }}=76 \mathrm{~cm}^{2} \approx|\widehat{\Sigma}|$. Moreover, we resample the noised measurements $Z_{n+1}$ in time with $\tau_{\text {obs }}=10 \tau=0.02 \mathrm{~s}$, and we re-interpolate linearly so that the filter can be applied at every time step of the simulation. The perfect signals and the noise (without time resampling for the sake of clarity) are shown in Figure 3 for representative points of the five regions of the vessel. Note that the signal-to-noise ratio decreases when going from the center to the ends of the AAA.

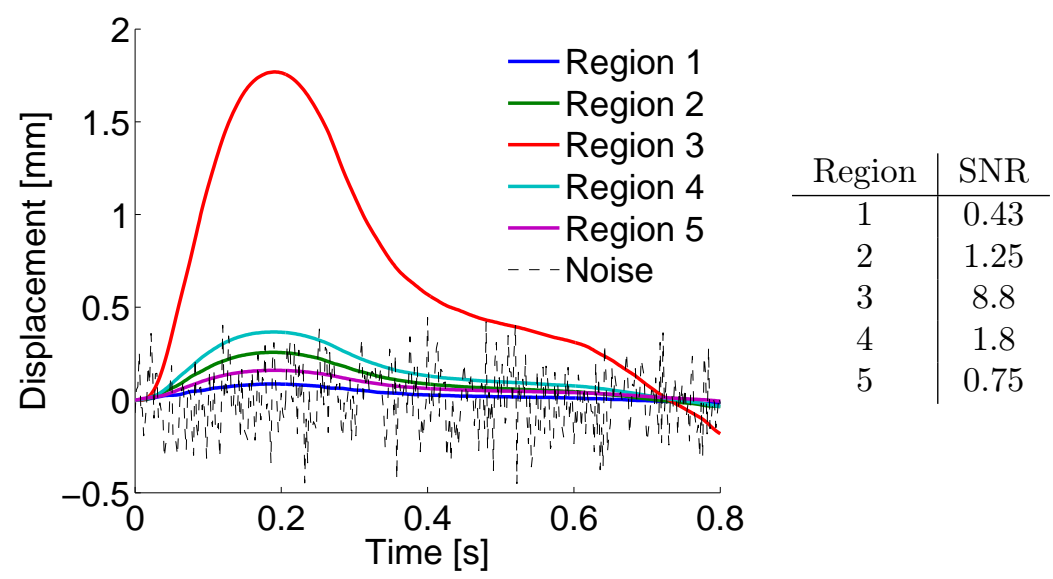

Figure 3: Noise compared to the typical wall displacements in the five regions. Time responses (left), and signal-to-noise ratio (SNR: signal mean divided by standard deviation) (right).

First estimation results In Figure 4, we present two estimation results for $E_{i}=2^{\theta_{i}}$ obtained for two different covariances $\alpha=4$ and $\alpha=9$ for $\theta_{i}$ in (49). We remind that the higher $\alpha$ the lower the confidence in the initial values of the parameters. The constant $\beta$ in 47 is for the moment fixed to 1 , which corresponds to a rather low confidence in the measurements.

Several comments are in order. First, comparing the two graphs of Figure 4 we observe that the estimation algorithm has more freedom to adapt the parameters for large values of $\alpha$. Second, the result is very good in region 3 but seems poor in the other regions. This is of course a consequence of the low SNR 
and the low value of $\beta$. Nevertheless, even in those cases, the algorithm is able to distinguished regions with high and low stiffness. This can be considered as a promising result for applications like detection of arteriosclerosis plaques from very noisy measurements.
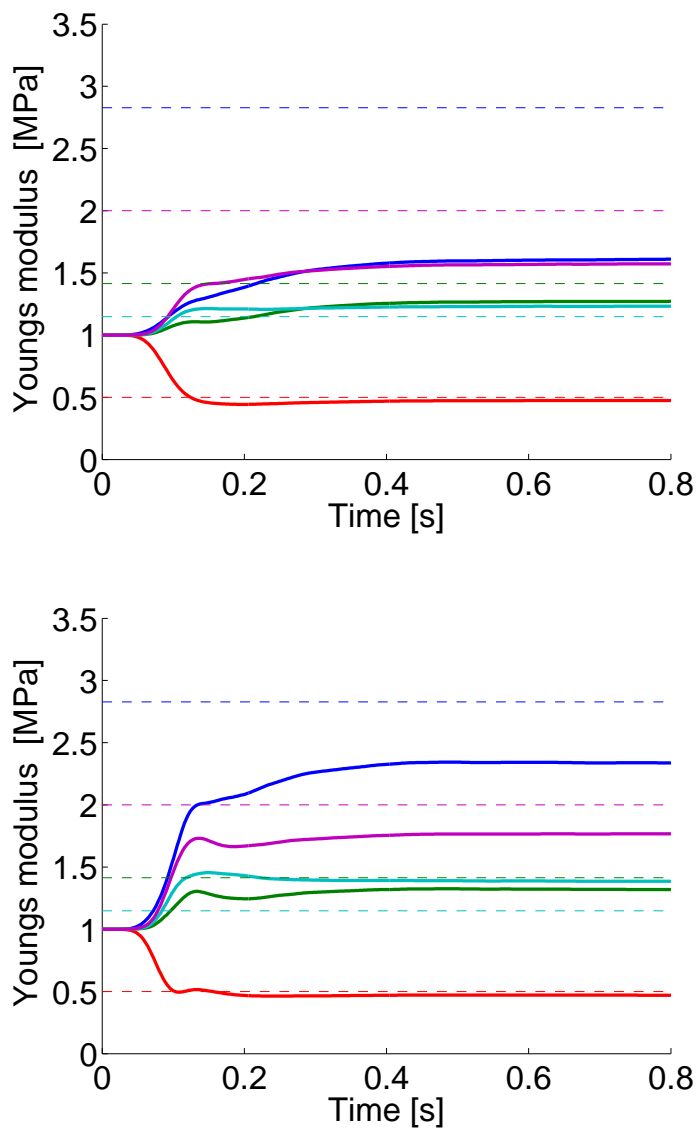

Figure 4: Results for the estimation of the Young's modulus with the reduced $\mathrm{UKF}$ algorithm for $\alpha=4$ (left) and $\alpha=9$ (right) for $\beta=1$. The dashed lines correspond to the correct values. The color are the same as in Figure 3

Sensitivity with respect to $\beta$ The results presented in Figure 4 can be improved by increasing the value of $\beta$, which means increasing our confidence in the measurements. Figure 5 shows the behavior of the estimated parameter at the end of the cardiac cycle when $\beta$ varies.

As expected, the sensitivity to $\beta$ is higher for large a priori covariances $\operatorname{Cov}\left(\zeta^{\theta}\right)=\alpha \mathbb{1}$, and the estimated value is much more sensitive to $\alpha$ in region 1 , which has the poorest SNR. But we also observe that for $\beta$ reasonably large (about 10), the values of $E$ are correctly estimated in all the other regions. Hence, at least for this example, we can conclude that, when the noise is not too 
high, the estimation does not strongly depend on the user-defined parameters $\alpha$ and $\beta$.
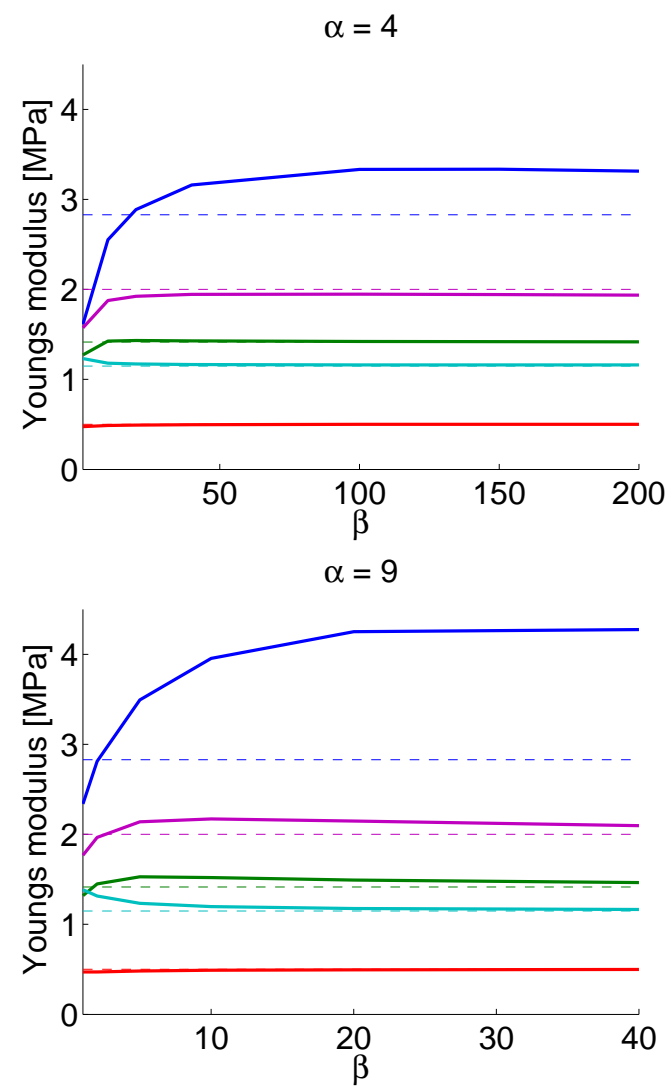

Figure 5: Results for the estimation of the Young's modulus (at $t=0.8$ ) with the reduced UKF algorithm for $\alpha=4,9$, for each section and different values of $\beta$. The relation color-region is the same as in Figure 3 . The dashed lines represents the reference values.

Estimation of the covariance The results presented so far corresponds to the estimated mean value of the parameters. An important feature of Kalman filtering is to also provide the estimated covariances, which is a valuable information about the confidence we can have in the results.

In the reduced UKF framework, the a posteriori covariance of the parameters is given by

$$
P_{n+1}^{\theta}=L_{n+1}^{\theta} U_{n+1}^{-1}\left(L_{n+1}^{\theta}\right)^{T}
$$

Figure 6 shows the results for the parameters mean $\hat{\theta}_{n}$ and mean plus/minus the standard deviation $\hat{\theta}_{n} \pm \sqrt{\operatorname{diag}\left(P_{n}^{\theta}\right)}$. For the sake of clarity, the results are presented here in terms of $\theta$ instead of $2^{\theta}$, and only for the worst and best regions ( 1 and 3 respectively). We observe that the a posteriori variance is much higher in the region where the parameter is poorly estimated. We are therefore 
informed by the algorithm that the results are less reliable in that case, even when the correct value is unknown. This point is particularly important when dealing with real data.
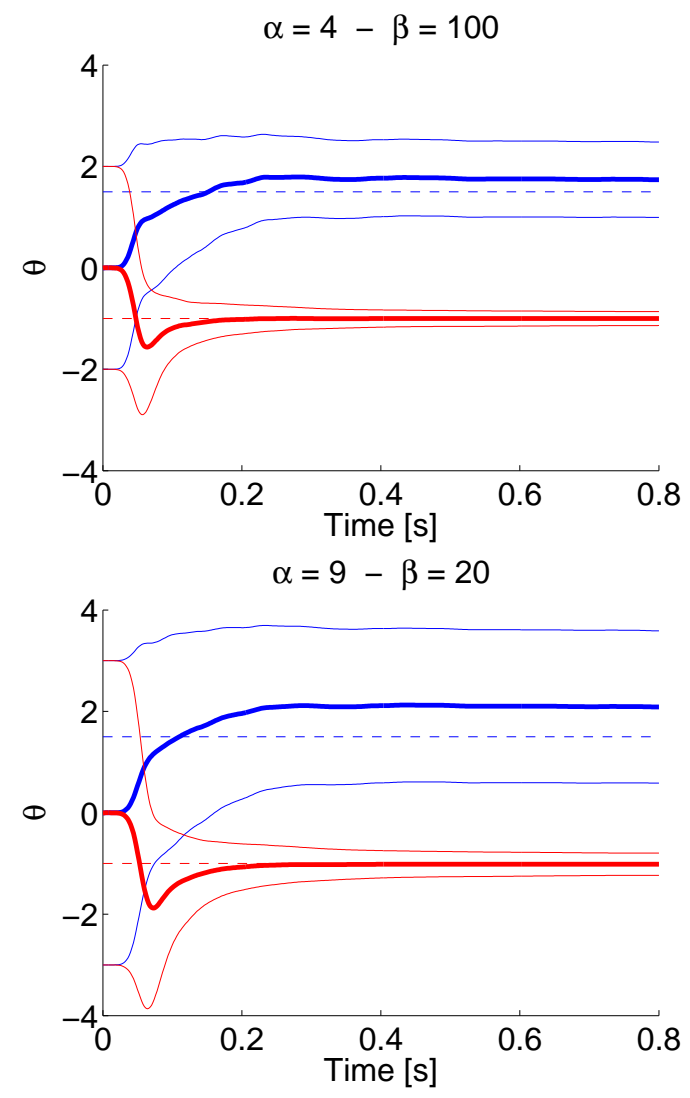

Figure 6: Results for $\hat{\theta}_{n}$ for Regions 1 (blue) and 3 (red) with $\alpha=4, \beta=100$ (left) and $\alpha=9, \beta=20$ (right) ( $\beta$ chosen in both cases to be in the flat region of figure 5). The thick continuos lines represent the mean value, the thin continous lines the mean plus/minus standard deviation, and the dashed lines the reference value as before.

\subsection{Estimation of the Windkessel's proximal resistance}

As indicated in section 4.2 , this estimation strategy does not involve many changes in the original software and can be implemented in a generic way outside the specific solvers. It is therefore quite simple to change or add parameters to estimate. In order to illustrate this versatility, we estimated the Windkessel's proximal resistance $R_{p}$ from the same measurements as before, i.e. synthetic noisy wall displacements. For this preliminary test, all the other parameters were supposed to be known. More complex situations will be considered in future works. As for the Young's modulus, we reparametrize $R_{p}$ as $R_{p}=500 \cdot 2^{\theta}$, and we perform the estimation on $\theta$. The result shown in Figure 7 was obtained 
with $\operatorname{Cov}\left(\zeta^{\theta}\right)=\alpha \mathbb{1}$, with $\alpha=1$. The value of $\beta=100$ was chosen so that the final estimation result is not sensible to it anymore. Note that can perfectly recover the reference value of $R_{p}$.

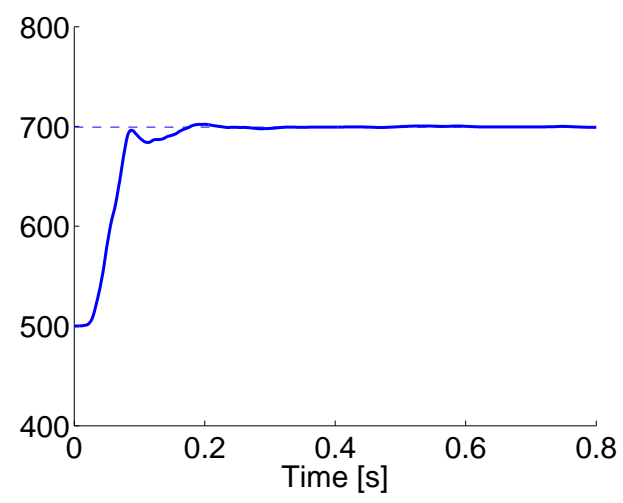

Figure 7: Results for the estimation of the proximal Windkessel resistance $\hat{R}_{p}$ with $\beta=100$.

\subsection{Error in the initial condition and filtering Windkessel's pressure}

Up to now, it has been assumed that the initial state is perfectly known. This is of course not the case in practice, and we would like to illustrate in this section the effect of an error in the initial condition. We will also show that the results can be improved by considering the Windkessel's pressure as a part of the filtered variables in the reduced UKF algorithm (we remind that up to now the filtered variables were restricted to the parameters).

Estimation with inexact initial condition To generate the inexact initial condition, the forward model is first run for one cardiac cycle with an homogeneous Young's modulus corresponding to $\theta_{i}=0$. The initial condition $\hat{X}_{0}$ used for the estimation corresponds to the state obtained at the end of the cycle $(t=0.8 s)$. Compared to the exact solution, the pressure difference is $2.4 \mathrm{mmHg}$ and the displacement difference is $0.16 \mathrm{~mm}$ in the AAA center (see Figure 8). The velocity and displacement differences in the fluid and the solid are negligible.

Figure 9 (left) shows the parameter estimation results obtained with $\alpha=4$, $\beta=100$ (the value of $\beta$ is chosen so that we are in the flat zone shown in Figure 5. Comparing with Figure 4, we see how the perturbation of the initial state deteriorates the estimation results. The pressure is lower due to the error in the initial condition (Figure 10, right, dashed cyan curve), which affects the parameter estimation.

As already mentioned, a first solution to address this problem is to filter the state with a physical Luenberger feedback, like in 24 for elastodynamics and 3] for FSI. The complexity of Luenberger filters remains moderate when the system 

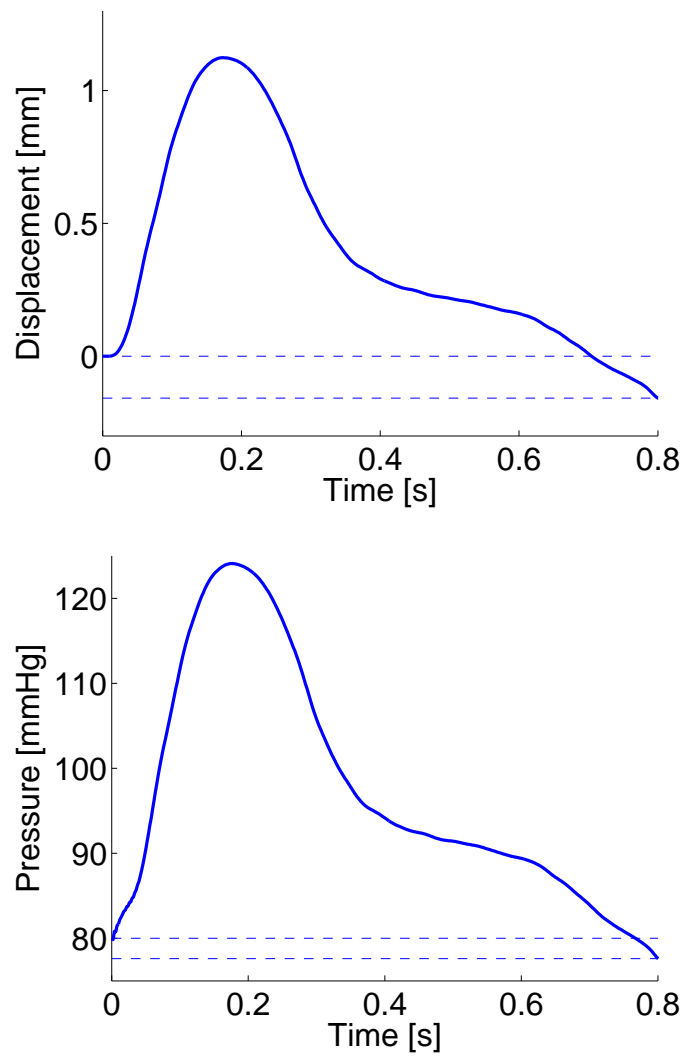

Figure 8: Response of the forward model with $\theta_{i}=0, \forall i$, for displacements at the AAA center (left) and proximal pressure $\bar{p}$ at $\Gamma^{\text {out }}$ (right) . The dashed lines show the gap between $t=0 \mathrm{~s}$ and $t=0.8 \mathrm{~s}$. 
dimension increases since it is based on regular mechanical stabilization terms. In the next section we propose a simpler solution, less general, but apparently quite efficient for blood flow simulations.

Filtering the Windkessel's pressure So far, the reduced order UKF algorithm has only been applied to the parameters. Now we propose to also apply it to the Windkessel's pressure $\pi$ which is a state variable of the problem. Contrary to the other state variables - the fluid and solid velocities and displacements, which depend on the spatial discretization $-\pi^{n}$ has by definition a limited size: just one real value in the experiment considered here, or a few real values in a case with several outlets. Therefore, it is straightforward to include it in the parameter space. From a practical viewpoint, this only consists of considering $\pi^{n}$ as a (time dependent) parameter in the above algorithm. If a new processor can be affected to this new "parameter", the computational cost remains almost unaffected. From the physical point of view, the fluid pressure is mainly governed by the Windkessel's pressure. Hence, improving the knowledge of this quantity would have a global impact on the estimation of the whole FSI system. Figure 9 (right) shows the estimation results in the case with an initial condition error (see the previous paragraph). We observe that the result is significantly improved.

Figure 10 shows the displacements and the pressure for different cases: reference (the solution to recover), not filtered, estimated with a perfectly known initial condition, estimated with error in the initial condition with and without filtering the Windkessel's pressure. Note that in all the filtered cases, the displacements are properly recovered. But we observe a substantial improvement in the pressure estimation when the Windkessel's pressure is filtered. This good estimation of the general amount of stress in the system, combined with the displacement accuracy, implies that the Young's modulus estimation is better than in the situation when $\pi^{n}$ is not filtered.

\section{Conclusions}

We have derived a procedure to estimate uncertain physical parameters in systems involving the mechanical interaction of a viscous incompressible fluid and an elastic structure. The method is based on the reduced-order Unscented Kalman Filter, introduced in [23. The algorithm does not need any tangent or adjoint problems and can easily be run in parallel, using as many processors as the number of parameters plus one. Doing so, the computational time needed to solve the inverse problem is of the same order as the time needed by a forward simulation using one processor.

Some numerical results have been presented for noised synthetic data corresponding to an idealized geometry of aneurysm. We have estimated the artery Young's modulus in 5 regions using measurements of the wall displacement. We have first considered a problem with a perfectly known initial condition, and we have investigated the sensitivity of the results to the noise and the a priori parameter covariance matrices. Then, we have perturbed the initial condition of the system in order to illustrate its impact on the parameter estimation. Finally, we have shown that considering the Windkessel's pressure as a parameter is an easy way to improve the estimation performance, without significantly 

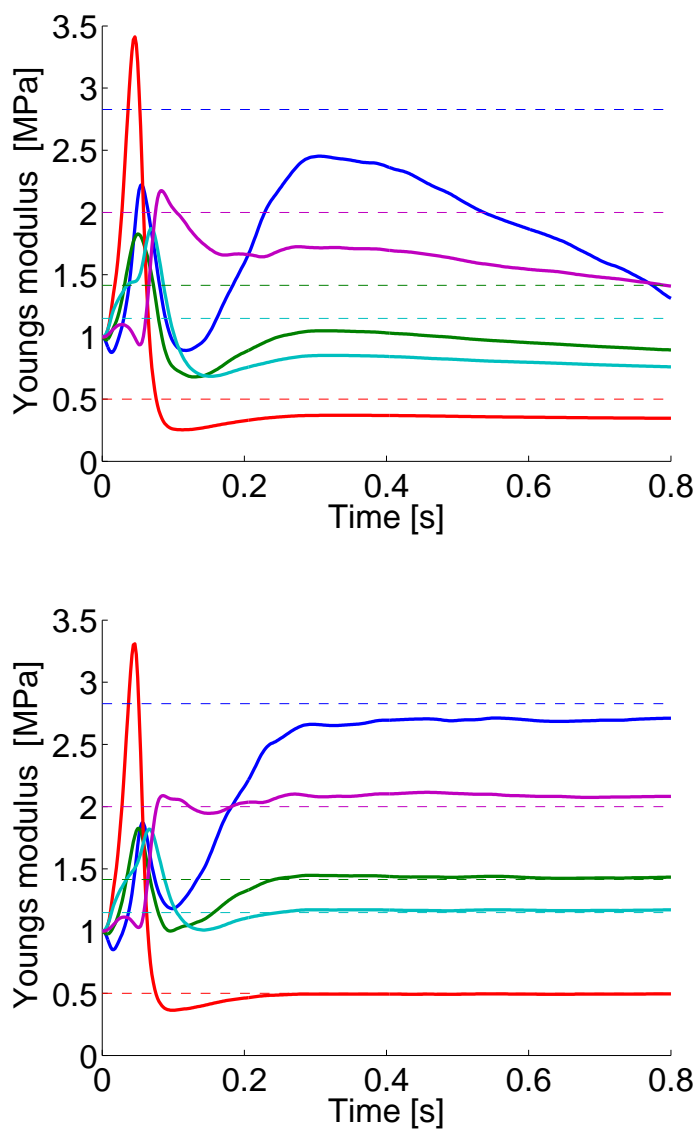

Figure 9: Parameter estimation in presence of an error in the initial condition with $\alpha=4, \beta=100$. Only 5 stiffness parameters included in the parameter space for the reduced UKF (left), and and effect of inclusion the Windkessel's pressure (right). 

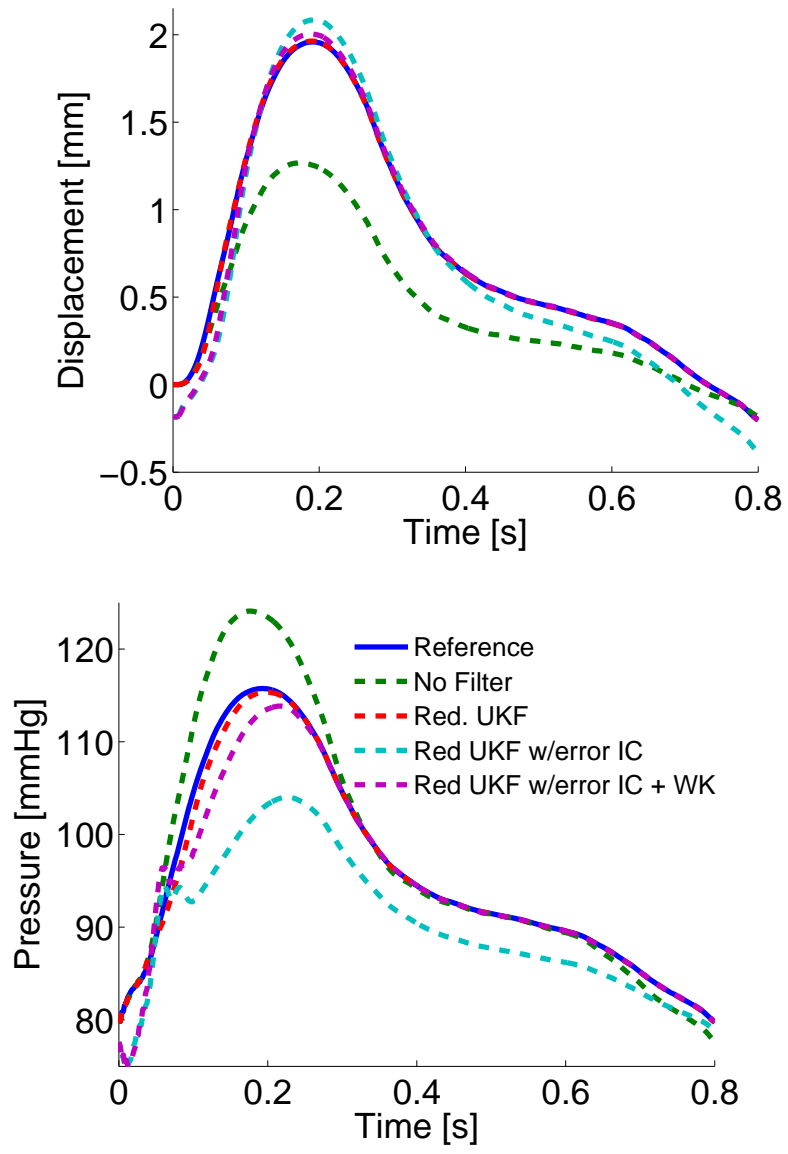

Figure 10: Results for the estimation of displacement at the AAA centre and Windkessel's pressure. All reduced UKF curves are obtained with $\alpha=4$ and $\beta=100$. 
increasing the computational cost, and without any additional measurements. To illustrate the versatility of the method, we have also presented a preliminary result about Windkessel resistance estimation.

Future works will include the estimation of other parameters of the problem. Other kinds of measurements will also be considered, in particular in the fluid, like those obtained by catheters or phase-contrast MRI, which should improve the global observability - or identifiability - of the system.

Acknowledgement - The authors wish to thank Dominique Chapelle and Miguel Fernández for fruitful discussions. The research leading to these results has received funding from the European Community's Seventh Framework Programme (FP7/2007-2013) under grant agreement nÂ̌r 224495 (euHeart project).

\section{A Simplex sigma-points}

In this work we used the so-called simplex sigma-points $I^{(i)}, r=p+1$, that correspond to vectors of zero mean and $\sqrt{p}$ covariance in $\mathbb{R}^{p}$. Together with the weights $\alpha_{i}$, they are computed recursively as (see [27, 13])

$$
\left[I_{1}^{*}\right]=\left(-\frac{1}{\sqrt{2 \alpha}}, \frac{1}{\sqrt{2 \alpha}}\right), \alpha=\frac{1}{p+1}=\alpha_{i} \forall i
$$

and

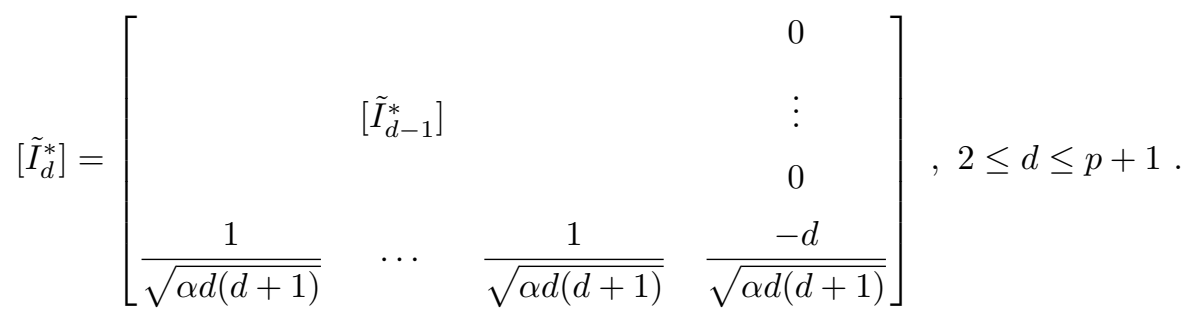

\section{References}

[1] H. T. Banks and K. Kunisch. Estimation techniques for distributed parameter systems, volume 1 of Systems \& Control: Foundations $\&$ Applications. Birkhäuser Boston Inc., Boston, MA, 1989.

[2] A. Bensoussan. Filtrage optimal des systèmes linéaires. Dunod, 1971.

[3] C. Bertoglio, D. Chapelle, M.A. Fernández, J-F. Gerbeau, and P. Moireau. Luenberger-based state estimation in vascular fluid-structure interaction through measurements in the solid. In preparation, 2011.

[4] C. Bertoglio, M.A. Fernández, J-F. Gerbeau, and Ph. Moireau. Filteringbased data assimilation in fluid-structure interaction: towards personalization of vascular models. In P.Nithiarasu and R.Löhner, editors, 1st International Conference on Mathematical and Computational Biomedical Engineering - CMBE, 2009. 
[5] G. Chavent. Nonlinear least squares for inverse problems. Scientific Computation. Springer, New York, 2009. Theoretical foundations and step-by-step guide for applications.

[6] M. D'Elia, L. Mirabella, T. Passerini, M. Perego, M. Piccinelli, C. Vergara, and A. Veneziani. Applications of variational data assimilation in computational hemodynamics. Technical Report TR-2011-002, Emory University, 2011.

[7] G. Evensen. Data assimilation: the ensemble Kalman filter. Springer Verlag, 2009

[8] M.A. Fernández. Coupling schemes for incompressible fluid-structure interaction: implicit, semi-implicit and explicit. Bol. Soc. Esp. Mat. Apl., to appear, 2011.

[9] M.A. Fernández and J-F. Gerbeau. Fluid structure interaction problems in haemodynamics, chapter 9. Springer Verlag, 2009. in Cardiovascular Mathematics. Modeling and simulation of the circulatory system, L. Formaggia, A. Quarteroni, A. Veneziani editors.

[10] M.A. Fernández, J-F. Gerbeau, and C. Grandmont. A projection algorithm for fluid-structure interaction problems with strong added-mass effect. $C$. R. Math. Acad. Sci. Paris, 342(4):279-284, 2006.

[11] M.A. Fernández, J.F. Gerbeau, and C. Grandmont. A projection semiimplicit scheme for the coupling of an elastic structure with an incompressible fluid. Int. J. Num. Meth. Engrg., 69(4):794-821, 2007.

[12] L. Formaggia, A. Quarteroni, and A. Veneziani, editors. Cardiovascular Mathematics. Modeling and simulation of the circulatory system, volume 1 of Modeling, Simulation and Applications. Springer, 2009.

[13] I. Hoteit, D.-T. Pham, and J. Blum. A simplified reduced order Kalman filtering and application to altimetric data assimilation in Tropical Pacific. Journal of Marine Systems, 36(1-2):101-127, 2002.

[14] S. Julier. The spherical simplex unscented transformation. In Proc. of the American Control Conference, pages 2430-4234, 2003.

[15] S. Julier, J. Uhlmann, and H.F. Durrant-Whyte. A new approach for filtering nonlinear systems. In American Control Conference, pages 1628$1632,1995$.

[16] S. Julier, J. Uhlmann, and H.F. Durrant-Whyte. A new method for the nonlinear transformation of means and covariances in filters and estimators. Automatic Control, IEEE Transactions on, 45(3):477-482, 2000.

[17] T. Kailath, A.H. Sayed, and B. Hassibi. Linear estimation. 2000.

[18] R. Kalman and R. Bucy. New results in linear filtering and prediction theory. Trans. ASME J. Basic. Eng, 83:95-108, 1961.

[19] P.-Y. Lagrée. An inverse technique to deduce the elasticity of a large artery. Eur. Phys. J. AP, 9:153-163, 1999. 
[20] D. G. Luenberger. An introduction to observers. IEEE Transactions on Automatic Control, 16:596-602, 1971.

[21] V. Martin, F. Clément, A. Decoene, and J-F. Gerbeau. Parameter identification for a one-dimensional blood flow model. ESAIM-Proceedings, $14: 174-200,2005$.

[22] P. Moireau and D. Chapelle. Erratum of article "reduced-order Unscented Kalman Filtering with application to parameter identification in large-dimensional systems". COCV, 17:406-409, 2011. doi:10.1051/cocv/2011001.

[23] P. Moireau and D. Chapelle. Reduced-order Unscented Kalman Filtering with application to parameter identification in large-dimensional systems. COCV , 17:380-405, 2011. doi:10.1051/cocv/2010006.

[24] P. Moireau, D. Chapelle, and P. Le Tallec. Joint state and parameter estimation for distributed mechanical systems. Computer Methods in Applied Mechanics and Engineering, 197:659-677, 2008.

[25] P. Moireau, D. Chapelle, and P. Le Tallec. Filtering for distributed mechanical systems using position measurements: Perspective in medical imaging. Inverse Problems, 25(3):035010-035035, March 2009.

[26] P. Moireau, N. Xiao, M. Astorino, C. A. Figueroa, D. Chapelle, C. A. Taylor, and J-F. Gerbeau. External tissue support and fluid-structure simulation in blood flows. Biomechanics and Modeling in Mechanobiology, 2011. doi: 10.1007/s10237-011-0289-z.

[27] D.-T. Pham, J. Verron, and L. Gourdeau. Filtres de Kalman singuliers évolutifs pour l'assimilation de données en océanographie. Comptes Rendus de l'Académie des Sciences - Series IIA, 326(4):255-260, 1998.

[28] D. Simon. Optimal state estimation: Kalman, H infinity, and nonlinear approaches. Wiley-Interscience, 2006.

[29] D. Simon. Kalman filtering with state constraints: A survey of linear and nonlinear algorithms. IET Control Theory \& Applications, accepted for publication, 2009.

[30] D. Tuan Pham, J. Verron, and M. Christine Roubaud. A singular evolutive extended kalman filter for data assimilation in oceanography. Journal of Marine systems, 16(3-4):323-340, 1998.

[31] J. Xi, P. Lamata, J. Lee, P. Moireau, D. Chapelle, and N. Smith. Myocardial transversely isotropic material parameter estimation from in-silico measurements based on reduced-order unscented kalman filter. Journal of the Mechanical Behavior of Biomedical Materials, 2011. 


\section{Contents}

$\begin{array}{lll}1 & \text { Introduction } & 3\end{array}$

2 Problem setting 4

2.1 Fluid-Structure model . . . . . . . . . . . . . . . . . . . . . . . . 4

2.2 Data assimilation problem . . . . . . . . . . . . . 5

\begin{tabular}{llll}
\hline 3 & Kalman filtering in a nutshell & $\mathbf{7}$
\end{tabular}

$3.1 \quad$ Static linear case: least squares estimation . . . . . . . . . . . . . 8

3.2 Dynamic linear case: the Kalman filter . . . . . . . . . . . . . . . 8

3.3 Extensions to nonlinear cases . . . . . . . . . . . . . . . . . 99 9

3.4 Unscented transforms and application to Kalman filtering . . . . 10

3.5 Factorized formulation of the UKF . . . . . . . . . . . . . . 12

$3.6 \quad$ Reduced-order UKF for parameter estimation . . . . . . . . . . . . 13

4 Reduced Order filtering for FSI problems

4.1 Fluid-Structure algorithm . . . . . . . . . . . . . . . . . 15

4.2 Algorithmic aspects of FSI filtering . . . . . . . . . . . . . . 17

$\begin{array}{lll}5 & \text { Numerical examples } & \mathbf{1 8}\end{array}$

$5.1 \quad$ Forward model description . . . . . . . . . . . . . . . . . . . . . . 18

5.2 Estimation of the Young's modulus . . . . . . . . . . . . . . . . . 19

5.3 Estimation of the Windkessel's proximal resistance . . . . . . . . 23

5.4 Error in the initial condition and filtering Windkessel's pressure. 24

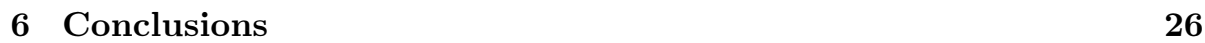

\begin{tabular}{|lr|}
\hline A Simplex sigma-points & 29
\end{tabular} 
Centre de recherche INRIA Paris - Rocquencourt Domaine de Voluceau - Rocquencourt - BP 105 - 78153 Le Chesnay Cedex (France)

Centre de recherche INRIA Bordeaux - Sud Ouest : Domaine Universitaire - 351, cours de la Libération - 33405 Talence Cedex Centre de recherche INRIA Grenoble - Rhône-Alpes : 655, avenue de l'Europe - 38334 Montbonnot Saint-Ismier Centre de recherche INRIA Lille - Nord Europe : Parc Scientifique de la Haute Borne - 40, avenue Halley - 59650 Villeneuve d'Ascq Centre de recherche INRIA Nancy - Grand Est : LORIA, Technopôle de Nancy-Brabois - Campus scientifique 615, rue du Jardin Botanique - BP 101 - 54602 Villers-lès-Nancy Cedex

Centre de recherche INRIA Rennes - Bretagne Atlantique : IRISA, Campus universitaire de Beaulieu - 35042 Rennes Cedex Centre de recherche INRIA Saclay - Île-de-France : Parc Orsay Université - ZAC des Vignes : 4, rue Jacques Monod - 91893 Orsay Cedex Centre de recherche INRIA Sophia Antipolis - Méditerranée : 2004, route des Lucioles - BP 93 - 06902 Sophia Antipolis Cedex 